\title{
YVES GUIVARC'H
}

\section{Marches aléatoires sur les groupes}

Publications de l'Institut de recherche mathématiques de Rennes, 1996-1997, fascicule 2 « Fascicule de probabilités », , p. 1-37

<http://www.numdam.org/item?id=PSMIR_1996-1997__2_A3_0>

(C) Département de mathématiques et informatique, université de Rennes, 1996-1997, tous droits réservés.

L'accès aux archives de la série «Publications mathématiques et informatiques de Rennes » implique l'accord avec les conditions générales d'utilisation (http://www.numdam.org/conditions). Toute utilisation commerciale ou impression systématique est constitutive d'une infraction pénale. Toute copie ou impression de ce fichier doit contenir la présente mention de copyright.

\section{Numdam}

Article numérisé dans le cadre du programme Numérisation de documents anciens mathématiques http://www.numdam.org/ 


\title{
MARCHES ALÉATOIRES SUR LES GROUPES
}

\author{
Yves GuIVARC'H
}

IRMAR, Université de Rennes 1, Campus de Beaulieu, 35042 RENNES Cedex

Ce sujet a été motivé par des questions variées de Calcul des Probabilités, de Théorie des groupes, d'Analyse harmonique, de Théorie ergodique et de Physique statistique. On mentionne quelques problèmes à l'origine de son développement, puis on les situe dans un cadre probabiliste général. On précise dans quelques directions les résultats obtenus et les problèmes qui restent posés. Enfin on esquisse les principaux développements actuels.

\section{I - Quelques problèmes et exemples classiques.}

1) Le battage des cartes et le "principe ergodique".

Considérons un jeu de $r$ cartes noté $J$ dont on suppose qu'il possède un ordre privilégié $\{1,2, \ldots, r\}$. Lors d'un battage de cartes cet ordre est changé en $\{\sigma(1), \sigma(2), \ldots, \sigma(r)\}$ où $\sigma$ est une permutation appartenant au groupe $\Sigma$ des permutations de $r$ objets. Si l'ordre initial était différent de $\{1,2, \ldots, r\}$, par exemple $\left\{a_{1}, a_{2}, \ldots a_{r}\right\}$ le même battage donnerait le nouvel ordre $\left\{\sigma\left(a_{1}\right), \sigma\left(a_{2}\right)\right.$, $\left.\ldots, \sigma\left(a_{r}\right)\right\}$. Un battage donné est donc représenté par une permutation $\sigma \in \Sigma$. Les habitudes du joueur sont telles qu'il choisit un certain nombre de permutations $\alpha \in A \subset \Sigma$ (éventuellement toutes) avec certaines probabilités fixées $p(\alpha)(\alpha \in A$, $\left.\sum_{\alpha \in A} p(\alpha)=1\right)$. Losqu'il effectue un second battage, il ne tient pas compte du premier et choisit donc de manière indépendante encore une permutation avec la même probabilité. La position probable de la ième carte après le premier battage est donc représentée par la répartition $\mu^{i}(1)=\sum_{\alpha \in A} p(\alpha) \delta_{\alpha(i)}$.

Après le deuxième battage cette répartition devient $\mu^{i}(2)=\sum_{(\alpha, \beta) \in A \times A} p(\beta) p(\alpha) \delta_{\beta \alpha(i)}$ où $\beta \alpha$ est la composée des permutations $\beta$ et $\alpha$.

Après $n$ battages on obtient la répartition $\mu^{i}(n)=\sum_{\left(\alpha_{1}, \alpha_{2}, \ldots, \alpha_{n}\right) \in A^{n}} p\left(\alpha_{n}\right) \ldots p\left(\alpha_{1}\right) \delta_{\alpha_{n} \ldots \alpha_{1}(i)}$. Notant $p^{n}(\sigma)=\sum_{\left(\alpha_{n} \ldots \alpha_{1}\right)=\sigma} p\left(\alpha_{n}\right) \ldots p\left(\alpha_{1}\right)$ on peut simplifier cette écriture en : $\mu^{i}(n)=\sum_{\sigma \in \Sigma} p^{n}(\sigma) \delta_{\sigma(i)}$. 
Le problème du battage des cartes consiste à montrer que, sous des hypothèses de non dégénérescence de $p$ la répartition $\mu^{i}(n)$ converge si $n$ tend vers l'infini, vers la répartition uniforme $\mu=\frac{1}{r} \sum_{j \in J} \delta_{j}$.

Sous une deuxième forme il consiste à répondre à la question analogue où $J$ est remplacé par $\Sigma$ lui-même. Si on ne s'intéresse pas à une carte fixée $i$ mais à l'ensemble du jeu, la répartition de celui-ci à l'instant $n$ est représentée par la mesure $\operatorname{sur} \Sigma$ :

$$
\mu_{n}=\sum_{\sigma \in \Sigma} p^{n}(\sigma) \delta_{\sigma}
$$

Dans cette écriture un ordre du jeu est assimilé à une permutation $\sigma$. La question est alors celle de la convergence de $\mu_{n}$ vers la répartition uniforme $m$ sur $\Sigma$, qui ne privilégie aucun ordre particulier : $m=\frac{1}{r !} \sum_{\sigma \in \Sigma} \delta_{\sigma}$.

Ce problème a été examiné de manière détaillée, par diverses méthodes par de nombreux auteurs en particulier H. Poincaré [P, p 301-313] et J. Hadamard [H]. Sous des conditions simples concernant la mesure $p$ la réponse à ces deux questions est positive et il y a de plus une vitesse de convergence exponentielle vers l'état d'équilibre $\mu$ ou $m$. La méthode de $\mathrm{H}$. Poincaré utilise la théorie des représentations des groupes finis, tandis que la plupart des auteurs utilisent les propriétés de l'itération d'une matrice positive. Ces méthodes ont été reprises récemment, pour une étude beaucoup plus approfondie par P. Diaconis en particulier [Di]. Cette question a suscité l'intérêt de mathématiciens éminents car elle met en évidence, sous une forme particulièrement simple, le "principe ergodique" dont la validité paraissait essentielle pour la justification de la mécanique statistique après les travaux de Gibbs et de Boltzmann.

Ce principe comporte dans ce cas particulier plusieurs aspects. D'abord l'oubli des conditions initiales, en ce sens que l'état d'équilibre limite est indépendant de l'ordre initial du jeu ; c'est l'indépendance mutuelle des différents battages qui conduit à un tel résultat. Un deuxième aspect important est l'indépendance du résultat final par rapport aux habitudes du joueur représentées par la probabilité $p$. Un troisième aspect est que la répartition limite est la mesure de probabilité $m$ sur $\Sigma$ "d'entropie maximale", c'est-à-dire la plus désordonnée possible. Rappelons que l'entropie d'une mesure de probabilité $v$ sur un ensemble fini $E$ est donnée par

$$
h(v)=\sum_{e \in \Sigma}-v(e) \log v(e) .
$$


Elle est nulle si $v$ est concentrée en un point et toujours inférieure ou égale au logarithme du cardinal de $E$, l'égalité n'étant réalisée que pour la mesure uniforme sur $E$.

2) La marche au hasard élémentaire dans $\mathbb{Z}^{d}$ et son interprétation en termes de réseaux électriques : transience et récurrence.

Dans le jeu de pile ou face, avec une "pièce non biaisée" les deux résultats $P$ et $F$ sont également possibles et les tirages successifs sont indépendants. On est alors conduit à attribuer à chaque suite de $n$ résultats la même probabilité $\frac{1}{2^{n}}$. Ces suites finies de $n$ éléments $P$ ou $F$ forment un ensemble $\Omega_{n}$ à $2^{n}$ éléments qui est muni d'une probabilité $\pi_{n}$. En fait, comme le nombre de tirages est non borné on doit considérer l'espace $\Omega=\Omega_{\infty}=\{P, F\}^{N}$ des suites illimitées en $P$ et $F$. Il et alors possible de définir sur $\Omega_{\infty}$ une structure d'espace métrique compact et une unique mesure de probabilité $\pi$ dont les projections sur les espaces $\Omega_{n}$ sont les probabilités $\pi_{n}$. C'est la mesure produit $\pi=p^{N}$ avec $p=\frac{1}{2}\left(\delta_{P}+\delta_{F}\right)$; la notion d'ensemble négligeable, (par rapport à $\pi$ ) ou de probabilité nulle acquiert une signification. On notera $\omega$ un point de $\Omega$ et $\omega_{i}$ sa iième coordonnée qui vaut donc $P$ ou $F$.

On note $\varepsilon_{i}$ le gain du joueur au ì̀me tirage ; par exemple $\varepsilon_{i}=1$ si $\omega_{i}=P$ et $\varepsilon_{i}=-1$ si $\omega_{i}=F$. Pour un tirage $\omega \in \Omega$ on peut alors considérer le gain total $S_{n}(\omega)$ du joueur jusqu'à l'instant $n: S_{n}(\omega)=\sum_{i=1}^{n} \varepsilon_{i}$.

Dans ce cadre il est bien connu que, avec probabilité 1 , le gain $S_{n}(\omega)$ s'annule pour une infinité d'entiers [F].

De manière analogue, on peut considérer le réseau $\mathbb{Z}^{d}$ de base canonique $e_{i}$ $(1 \leq i \leq d)$, la mesure de probabilité $p=\frac{1}{2 d} \sum_{i=1}^{d}\left(\delta_{e_{i}}+\delta_{-e_{i}}\right)$.

Une marche "au hasard" sur $\mathbb{Z}^{d}$ se trouve alors définie de la façon suivante : si à l'instant $n$ le marcheur se trouve en $x_{n} \in \mathbb{Z}^{d}$, à l'instant $n+1$ il se trouvera en l'un des points voisins $x_{n+1}=x_{n} \pm e_{i}$ avec probabilité $\frac{1}{2 d}$. Si on note $\varepsilon_{k}= \pm e_{i}$ le pas choisi à l'instant $k$, sa position à l'instant $n$, si au temps 0 on a $x_{0}=0$, sera donnée par $x_{n}(\omega)=S_{n}(\omega)=\sum_{i=1}^{n} \varepsilon_{i} \in \mathbb{Z}^{d}$. 
Ici $\omega \in \Omega=\left\{ \pm e_{i}, 1 \leq i \leq d\right\}^{N}$ est la suite des pas choisis et comme dans le jeu de pile ou face une probabilité naturelle $\pi=p^{N}$ est définie sur $\Omega$ : la probabilité de l'ensemble des suites commençant par $n$ symboles fixés est $\left(\frac{1}{2 d}\right)^{n}$.

Une question se pose : quelle est la probabilité du marcheur de revenir à l'origine. Pour $d=2$, c'est le problème dit du marcheur de Manhattan qui, à chaque carrefour choisit au hasard sa direction ; on demande si, partant de chez lui il y reviendra après un certain nombre de pas (non fixé à l'avance). La réponse est positive avec probabilité 1 . Avec probabilité 1 , il y aura également une infinité de retours à l'origine. Au contraire pour $d>2$, la probabilité d'un retour est inférieur à 1 et celle d'une infinité de retours est nulle [Sp].

Ces résultats sont dus à Polya et illustrent le rôle critique de la dimension 2 dans le problème de la marche au hasard sur $\mathbb{Z}^{d}$. Ils admettent une interprétation en termes de réseaux électriques fort intéressante, qui conduit à de nouvelles démonstrations à l'aide de la notion d'énergie et du "principe de Dirichlet". Cette connection semble avoir été remarquée pour la première fois par Nash et Williams [N-W]. On peut la décrire brièvement ainsi [voir Do-S]. On attribue à chaque arête de $\mathbb{Z}^{d}$ la résistance 1 et on suppose valides les lois de l'électricité pour ce réseau de résistances. On demande en particulier quelle est la résistance effective $R_{e f}$ du réseau lorsqu'une différence de potentiel unité est appliquée entre 0 et l'infini de $\mathbb{Z}^{d}$.

La réponse générale est que le produit de $R_{e f}$ par la probabilité de non retour à l'origine est un nombre strictement positif. En particulier $R_{e f}$ est finie si et seulement si $d>2$. Pour $d \leq 2$, la résistance effective est infinie, ce qui s'interprête physiquement comme l'absence de courant électrique (d'énergie finie) entre 0 et l'infini. L'assertion contraire est vraie pour $d>2$.

Si l'on note $\Delta$ le "laplacien discret" défini sur $\mathbb{Z}^{d}$ par

$$
(\Delta f)(x)=\frac{1}{2 d}\left[\sum_{i=1}^{d} f\left(x+e_{i}\right)+f\left(\left(x-e_{i}\right)\right]-f(x) .\right.
$$

Le "potentiel" $v(x)$ correspondant devrait satisfaire les conditions

$$
\begin{gathered}
v(0)=1 \quad, \Delta v(x)=0 \quad \text { si } x \neq 0 \\
\lim _{x \rightarrow \infty} v(x)=0
\end{gathered}
$$

où la deuxième équation correspond au fait que la somme des courants issus de $x$ est nulle. Le problème considéré ici appartient donc clairement à la théorie du potentiel. On observera que la probabilité d'atteindre 0 partant de $x$ satisfait les deux premières conditions. 
Du point de vue probabiliste, pour $d>2$, la marche au hasard est "transiente" en ce sens que le marcheur partant de l'origine aboutira à l'infini ; au contraire pour $d \leq 2$, ses retours successifs à l'origine permettent de qualifier la marche de "récurrente".

3) Une version simplifiée du problème de la longueur moyenne des polymères.

Un modèle de polymère plan simplifié est une ligne brisée $A_{0} A_{1} \ldots A_{n}$ où chaque segment $A_{i} A_{i+1}$ est de longueur 1 et l'angle de $\overrightarrow{A_{i-1} A_{i}}$ avec $\overrightarrow{A_{i} A_{i+1}}$ vaut $\pm \alpha(\alpha \in] 0,2 \pi\left[\right.$, le signe \pm 1 étant choisi au hasard avec probabilité $\frac{1}{2}$ [voir M. Kac (K)]. Si $\left(\overrightarrow{A_{k-1} A_{k}}, \overrightarrow{A_{k} A_{k+1}}\right)=\alpha \varepsilon_{k}$ et si $\overrightarrow{A_{0} A_{1}}$ est le premier vecteur de base du plan euclidien, ici identifié au corps des nombres complexes $\mathbb{C}$, le vecteur $\overrightarrow{A_{0} A_{n}}$ sera représenté par la somme des nombres complexes $e^{i \alpha S_{k}}$ où $S_{k}=\varepsilon_{1}+\varepsilon_{2}+\ldots+\varepsilon_{k}(1 \leq k \leq n-1)$.

On doit alors évaluer le module du nombre complexe $Z_{n}=1+\sum_{1}^{n-1} e^{i \alpha S_{k}}$ qui est l'affixe du point $A_{n}$, en moyenne pour tous les choix possibles des $\varepsilon_{k}$.

L'espace $(\Omega, \pi)$ introduit dans le jeu de pile ou face intervient donc ici également et il est commode d'estimer la "longueur quadratique moyenne"

$\ell_{n}=\left(\int_{\Omega}\left|Z_{n}\right|^{2} d \pi\right)^{1 / 2}$.

Il a été montré par le chimiste Eyring dans ce type de situation que si $n$ tend vers l'infini $\frac{\ell_{n}}{\sqrt{n}}$ tend vers une constante $C(\alpha)$ strictement positive, ce qui est une forme de la loi normale de Gauss. Le polymère est donc pour $n$ très grand de l'ordre de $\sqrt{n}$, ce qui traduit un phénomène de repliement sur lui-même du polymère. Ce modèle néglige l'interaction des molécules situées aux sommets $A_{i}$, interaction qui introduit une contrainte dite de "volume exclu" $\left\|A_{i} A_{j}\right\| \geq \varepsilon$ $>0$. Ce dernier modèle plus réaliste est très voisin de la "marche aléatoire autoévitante". Son étude est très difficile et peu développée actuellement. Il est conjecturé que la longueur moyenne du polymère est de l'ordre d'une puissance de $n$ supérieure à $\frac{1}{2}$.

4) L'équirépartition sur la sphère et ses aspects arithmétiques.

Le problème suivant a été considéré par V. Arnold et A. Krylov [A.K.] en relation avec le comportement asymptotique des solutions d'équations différentielles de la variable complexe. 
On se donne deux rotations $a$ et $b$ autour de l'origine dans l'espace euclidien et on considère leur action sur la sphère unité $S^{2}$ de centre $O \in \mathbb{R}^{3}$. On note $\lambda(a, b)$ un produit quelconque de ces rotations en nombre $n$. On note alors $|\lambda|=n$. Soit $p \in S^{2}$ et $\lambda(a, b) . p$ son transformé sous l'action de la rotation $\lambda(a, b)$. On s'intéresse à la répartition des points $\lambda(a, b) . p$ lorsque $|\lambda|=n$ tend vers l'infini. En d'autres termes on considère la suite de mesures sur $S^{2}$ $\mu_{n}^{p}=\frac{1}{2^{n}} \sum_{|\lambda|=n} \delta_{\lambda(a, b) . p}$

où $\delta_{x}$ désigne la masse Dirac en $x$. On demande alors si $\mu_{n}^{p}$ converge vers la mesure uniforme sur $S^{2}$. Ceci revient à dire que, pour toute partie $A \subset S^{2}$ de frontière négligeable on $\mathrm{a}$

$$
\lim _{n} \mu_{n}^{p}(A)=\lim _{n} \frac{1}{2^{n}} \#\{\lambda ; \lambda(a, b) . p \in A ;|\lambda|=n\}=\sigma(A)
$$

où $\sigma$ est la mesure uniforme sur $S^{2}$. On demande aussi, si cette convergence a lieu, quelle est la vitesse de convergence. La réponse à la première question est positive sous des conditions de non-dégénérescence simples concernant les deux rotations $a$ et $b$; en particulier leurs axes ne doivent pas coïncider. La réponse à la deuxième question n'est pas actuellement vraiment élucidée. Il y a cependant des résultats partiels remarquables. Par exemple si l'on considère le sous-groupe $H$ de rotations engendré par les 3 rotations autour des axes de coordonnées et d'angles $\frac{3 \pi}{5}$, et si $a, b$ appartiennent à $H$, la discrépance $\operatorname{Sup}_{p}\left|\sigma(A)-\mu_{n}^{p}(A)\right|$ tend vers 0 exponentiellement [L.P.S], [Lu], [Sa].

Ceci doit être comparé à la situation correspondante sur le cercle $S^{1}$ où cette convergence n'est jamais exponentielle si $\alpha$ est irrationnel. Cette propriété fournit la solution d'un problème célèbre de Banach-Ruskiewicz [B] : la mesure uniforme sur $S^{2}$ est-elle la seule forme linéaire sur $\mathbb{L} \infty\left(S^{2}\right)$ invariante par rotations ? La réponse est positive [Sa] ; au contraire sur $S^{1}$, il existe une infinité de telles formes linéaires.

On notera l'analogie formelle de ce problème avec celui du battage des cartes qui en est en quelque sorte une version finie. La parenté des deux situations apparaît plus nettement si comme en [Di] on considère simultanément tous les groupes de permutations de $n$ éléments où $n$ tend vers l'infini.

5) Les fonctions harmoniques positives pour la marche aléatoire élémentaire sur le groupe libre à deux générateurs : analogie avec la formule de Poisson dans le disque.

Considérons le groupe libre $\Gamma$ engendré par les deux générateurs $a$ et $b$. Un élément de $\Gamma$ est donc un mot (réduit) en $a, b, a^{-1}, b^{-1}$ sans succession de 
$a, a^{-1}$ ou $b, b^{-1}$. La longueur de $\gamma \in \Gamma$ est notée $|\gamma|$ et une distance naturelle $d$ est définie sur $\Gamma$ par $d\left(\gamma, \gamma^{\prime}\right)=\left|\gamma^{-1} \gamma^{\prime}\right|$. Le groupe $\Gamma$ peut être compactifié par l'adjonction à $\Gamma$ de l'ensemble $\partial \Gamma$ des "mots infinis" ; un tel mot infini $\xi$ est une suite $\xi \in\left\{a, b, a^{-1}, b^{-1}\right\}^{N}$ où la succession de $a, a^{-1}$ et $b, b^{-1}$ est interdite. La convergence d'une suite $\gamma_{n}$ vers $\xi \in \partial \Gamma$ revient à la coïncidence pour $n$ assez grand des $n$-débuts des suites $\gamma$ et $\xi$. Le graphe de $\Gamma$ a une structure arborescente homogène à 4 branches et il en découle aisément que l'on peut définir la fonction suivante sur $\Gamma \times \partial \Gamma$ :

$$
\beta(\gamma, \xi)=\lim _{x_{n} \rightarrow \xi}\left[d\left(\gamma, x_{n}\right)-d\left(e, x_{n}\right)\right] .
$$

Dans cette expression, pour $n$ assez grand $d\left(\gamma, x_{n}\right)-d\left(e, x_{n}\right)$ est constant et est la différence des distances de $\gamma$ et $e$ au "confluent" de $\gamma$ et $\xi$.

Considérons alors le 'Laplacien discret $\Delta$ défini par

$$
\Delta f(\gamma)=\frac{1}{4}\left[f(\gamma a)+f\left(\gamma a^{-1}\right)+f(\gamma b)+f\left(\gamma b^{-1}\right)\right]-f(\gamma)
$$

et les solutions positives de l'équation $\Delta f=0$.

On vérifie aisément que pour tout $\xi$ fixé, la fonction $h_{\xi}(\gamma)=e^{-\beta(\gamma, \xi)}=P(\gamma, \xi)$ vérifie $\Delta h_{\xi}=0$.

E.B. Dynkin et M. Malyutov ont montré que toutes les solutions positives $f$ de cette équation sont données par la formule intégrale [Dy-M]

$$
f(\gamma)=\int P(\gamma, \xi) d v(\xi)
$$

où $v$ est une mesure positive sur $\partial \Gamma$ qui est uniquement définie par $f$.

Ce résultat doit être comparé à celui que l'on obtient dans $\mathbb{Z}^{d}$ pour le Laplacien discret considéré en 2): les seules solutions positives de $\Delta f=0$ sont les constantes ; la propriété analogue est valide dans $\mathbb{R}^{d}$ pour les fonctions harmoniques positives solutions de $\sum_{i=1}^{d} \frac{\partial^{2} f}{\partial^{2} x_{i}}=0$.

Au contraire, dans le disque unité il existe des fonctions harmoniques positives non triviales. Elles sont données par :

$$
f(z)=\frac{1}{2 \pi} \int_{0}^{2 \pi} P(z, \theta) d v(\theta)
$$

où $P(z, \theta)=\frac{1-|z|^{2}}{\left|e^{i \theta}-z\right|^{2}}$ est le noyau de Poisson et $v$ est une mesure positive sur le bord $|z|=1$, uniquement définie par $f$. L'analogie entre $\Gamma$ et le disque est éclairée si l'on observe que le Laplacien de la métrique de Poincaré sur le disque 
est $\left(1-|z|^{2}\right)^{2} \frac{\partial^{2}}{\partial z \partial \bar{z}}$ et donc que les fonctions harmoniques relatives à ce Laplacien sont aussi les solutions de $\frac{\partial^{2} f}{\partial z \partial \bar{z}}=0$. Le groupe préservant la structure conforme du disque est isomorphe au groupe des matrices de déterminant $1, G=S \ell(2, \mathbb{R})$ et présente des analogies importantes avec $\Gamma$. Au contraire le groupe conforme de $\mathbb{C}$ est celui des similitudes $z \rightarrow a z+b$ ( $a \in \mathbb{C}^{*}, b$ $\in \mathbb{C}$ ). Le présence ou l'absence de fonctions positives harmoniques non triviales tient aux différences de structure entre ces groupes, ainsi que l'a remarqué H. Furstenberg [Fu1].

6) Un exemple de relation de récurrence à coefficients aléatoires : l'équation de Schroëdinger discrète en milieu aléatoire sur $\mathbb{Z}$.

Considérons dans $\mathbb{R}^{d}$ le Laplacien $\Delta$ et un potentiel $V(x)$ qui est une fonction réelle. L'opérateur de Schroëdinger associe à une fonction $u$ de classe $C^{2}$ la fonction $-\Delta u(x)+V(x) u(x)$; sous des conditions peu exigeantes concernant $V$, c'est un opérateur auto-adjoint dans $\mathbb{L}^{2}\left(\mathbb{R}^{d}\right)$. On est en particulier intéressé par l'équation dite stationnaire

$-\Delta u+V u=\lambda u$

où $\lambda \in \mathbb{R}$ et on demande s'il existe une base orthonormale de $\mathbb{L}^{2}\left(\mathbb{R}^{d}\right)$ formée de telles fonctions ; on dit alors que la particule est localisée ; la fonction $|u|^{2}$ est appelée probabilité de présence de la particule mais on ne s'intéresse pas ici aux relations de cette notion avec le calcul des probabilités. Dans les exemples analytiques la localisation est rarement réalisée et on s'intéresse alors au spectre de l'opérateur de Schroëdinger qui contient lui aussi des informations intéressantes sur le phénomène physique considéré. Ici on remplace l'opérateur $-\Delta+V$ par l'opérateur discrétisé correspondant sur $\mathbb{Z}^{d}$.

Une question posée par les célèbres physiciens Mott et Anderson au début des années 60 est de savoir si dans $\mathbb{Z}^{3}$, il y a localisation lorsque le potentiel $V_{n}$ ( $n \in$ $\mathbb{Z}^{3}$ ) est une famille de variables aléatoires indépendantes de même loi. Dans cette généralité, le problème n'est pas actuellement résolu malgré d'importantes contributions. La propriété d'indépendance des variables $V_{n}(\omega)$ traduit le désordre du milieu et celui-ci est susceptible de s'opposer à la propagation de la particule, donc de conduire à sa localisation.

Une version du problème en dimension 1 peut se formuler de la façon suivante. Soit $\varepsilon_{n}(\omega)$ une suite de variables indépendantes bornées (par exemple le résultat d'un tirage à pile ou face) et $S$ l'opérateur borné de $\ell^{2}(\mathbb{Z})$ défini par 
$(S u)_{n}=-\left(u_{n+1}+u_{n-1}\right)+\varepsilon_{n} u_{n}$.

Est-il vrai que pour presque tout $\omega$, l'opérateur auto-adjoint $S$ de $\ell^{2}(\mathbb{Z})$ admette une base de vecteurs propres ? Il a été montré par Goldcheid-Molcanov-Pastur [Go-M-P] que la réponse est positive sous des hypothèses de non-dégénérescence des $\varepsilon_{n}$, (voir par exemple $[C a-L]$ ). On est conduit à examiner la relation $\left(u_{n+1}+u_{n-1}\right)+\varepsilon_{n} u_{n}=\lambda u_{n}(\lambda \in \mathbb{R})$ c'est-à-dire sous forme matricielle

$$
\left(\begin{array}{c}
u_{n+1} \\
u_{n}
\end{array}\right)=\left(\begin{array}{cc}
\varepsilon_{n}-\lambda & -1 \\
0 & 1
\end{array}\right)\left(\begin{array}{c}
u_{n} \\
\\
u_{n-1}
\end{array}\right) \text {. }
$$

Si $A_{n}$ désigne la matrice $\left(\begin{array}{cc}\varepsilon_{n}-\lambda & -1 \\ 0 & 1\end{array}\right)$, qui appartient au groupe $S \ell(2, \mathbb{R})$, et $X_{n}$ le vecteur $\left(\begin{array}{c}u_{n} \\ \\ u_{n-1}\end{array}\right)$, on a donc la relation de récurrence $X_{n+1}=A_{n} X_{n}$ et en particulier

$$
X_{n}=A_{n-1} \ldots A_{0} X_{0}, \quad X_{n}=A_{n}^{-1} \ldots A_{-1}^{-1} X_{0} \quad(n \geq 0) \quad(n<0)
$$

Dans ce cas l'étude de la localisation peut se ramener à celle du produit de matrices indépendantes $S_{n}(\omega)=A_{n-1} \ldots . A_{0}(n \geq 0)$.

\section{II - Marches aléatoires sur les groupes.}

Les exemples de I suggèrent un cadre général d'étude faisant intervenir le calcul des probabilités, la théorie du potentiel, la théorie ergodique, la théorie des groupes. Une telle formalisation apparaît déjà dans l'ouvrage de P. Levy : "Les processus stochastiques et le mouvement brownien" [L, pp 175-187] dans le cadre des processus additifs dans les espaces euclidiens ou non euclidiens.

Dans les années 60, la théorie du potentiel sur les espaces discrets était déjà bien développée et a fourni un premier cadre d'étude, celui des marches aléatoires sur $\mathbb{Z}^{d}$ [cf. F. Spitzer, (Sp]. Des problèmes comme celui de l'équirépartition sur la sphère avaient déjà été étudiés par Kawada-Ito [Kaw-I] ; celui de la longueur des polymères signalés par $M$. Kac $[K]$ ou les résultats de P. Lévy cités précédemment, conduisent à envisager aussi des groupes non commutatifs. Nous en décrivons le cadre abstrait ci-dessous formulé dans les années soixante dix en généralisant directement le deuxième exemple de $\mathrm{I}$. 
Soit $G$ un groupe localement compact métrisable, $p$ une mesure de probabilité sur $G$. On appelle marche aléatoire droite de loi $p$, la chaîne de Markov d'espace d'états $G$ et de noyau de transition $P$ défini par

$$
P \varphi(g)=\int \varphi(g h) d p(h) \text {. }
$$

Les "transitions" sont donc ici assimilées à des "sauts aléatoires" à droite qui transforment $g$ en $g h$. On notera que $P$ commute avec l'action de translation à gauche, ce qui permet d'utiliser des méthodes d'Analyse harmonique. La mesure $v$ est transformée en $v P$ définie par $v P(\varphi)=\int \varphi(g h) d p(h) d v(g)$. En termes de convolution sur $G: v P=v * p$. En particulier si $v=m$ est la mesure de Haar à droite $m P=m * p=m$.

D'un point de vue probabiliste on est amené à considérer les trajectoires de la chaîne partant de $g$ :

$$
g S_{n}(\omega)=g x_{0}(\omega) \ldots x_{n-1}(\omega)
$$

où $\omega \in G^{N}$ a pour coordonnées $x_{i}(\omega) \in G$.

L'espace $\Omega$ est alors muni de la mesure produit $\pi=p^{N}$, comme dans le jeu de pile ou face et $\pi$ régit le choix de la suite de sauts indépendants $x_{i}(\omega) \quad(i \in N)$. L'espace des trajectoires est donc $\Omega \times G$ et le décalage $\hat{\theta}$ sur cet espace s'écrit

$$
\hat{\theta}(\omega, g)=\left(\theta \omega, g x_{0}(\omega)\right)
$$

où $\theta$ est le décalage sur $\Omega:(\theta \omega)_{i}=x_{i+1}(\omega)$.

La mesure $\pi \times \lambda$ est $\hat{\theta}$-invariante et l'on a la relation de cocycle à valeurs dans $G$, pour le système dynamique $(\Omega, \theta, \pi)$ :

$$
S_{m+n}(\omega)=S_{m}(\omega) S_{n} \circ \theta^{m}(\omega)
$$

De ce point de vue, le cadre probabiliste est très proche de la théorie ergodique et l'on peut, en principe, s'affranchir de l'hypothèse d'indépendance des $x_{i}(\omega)$ en la remplaçant par une hypothèse de stationnarité.

Enfin la théorie classique du potentiel a été motivée par la théorie de l'Électricité et on peut aussi donner une interprétation de la situation précédente dans ce cadre, du moins si $p$ est symétrique $(p=\check{p})$.

Les aspects physiques de la théorie de l'Électricité et du mouvement brownien paraissent très différents mais, au niveau des équations, ls opérateurs sui s'introduisent sont proches du laplacien. On peut donc tenter de tirer parti de cette double interprétation physique. Pour simplifier, on supposera ici que $G=\Gamma$ 
est discret et engendré par un ensemble fini de générateurs $V=\left\{a_{i}^{ \pm 1} ;(i=1, \ldots\right.$, $r)$ \}. On considère $\Gamma$ comme un graphe de sommets $\gamma \in \Gamma$ et d'arêtes $\gamma a_{i}^{ \pm 1}$ pondérées par les nombres $p\left(a_{i}\right)$.

Alors $\Gamma$ s'interprète comme un réseau (infini) de résistances électriques, le "noeud" autour de $\gamma$ étant formé des résistances $\left(\gamma, \gamma a_{i}^{ \pm 1}\right)$ de valeurs $\frac{1}{p\left(a_{i}\right)}$ (la conductance). On suppose alors valides les lois de Kirchoff et la loi d'Ohm et on peut demander quelle est la répartition des courants lorsque l'on fixe une différence de potentiel unité entre l'élément neutre de $\Gamma$ et l'infini. Du fait de l'introduction de l'infini dans ce problème de nature physique, on doit préciser et le lecteur pourra se reporter à l'ouvrage de Doyle et Snell [Do-S.]. Ce type de problème semble avoir été posé clairement pour la première fois par le topologue B. Eckmann [Eck] par analogie avec la théorie homologique. Par ailleurs plusieurs travaux de $\mathrm{H}$. Weyl [W] de cette époque mettent en évidence le rôle du Laplacien dans l'étude de l'homologie des variétés. En théorie du potentiel et en analyse, ce point de vue est très proche de celui des "méthodes hilbertiennes" développées par H. Cartan [C] et J. Deny [D].

Dans le cas d'une probabilité symétrique on peut donc utiliser les deux aspects de la théorie du potentiel : l'aspect "probabiliste" et l'aspect "électrique".

Après ces généralités, revenons brièvement aux exemples de I. Dans l'exemple du battage des cartes, le groupe considéré n'est autre que le groupe des permutations $\Sigma$ de l'ensemble fini et l'interprétation "probabiliste" comme chaîne de Markov sur un espace d'états fini est très naturelle. Cependant l'interprétation "électrique" conduit si $p=\check{p}$ à des inégalités non triviales [Di]. Dans l'exemple du jeu de pile ou face, le groupe est $\mathbb{Z}$ et la marche aléatoire va "aux plus proches voisins" avec égale probabilité. Il en est de même dans $\mathbb{Z}^{d}$ $(d>1)$ et l'interprétation "électrique" est très naturelle, par analogie avec le potentiel newtonien ou logarithmique. Ceci vaut aussi pour le groupe libre si on le considère comme plongé dans le disque hyperbolique ; la situation se rattache aussi bien au mouvement brownien qu'à la théorie du potentiel dans le disque. Dans le problème d'équirépartition sur la sphère et dans le problème de localisation, l'aspect ergodique est dominant et la non commutativité du groupe joue un rôle essentiel. Le problème de la longueur des polymères introduit la marche aléatoire sur le groupe des déplacements du plan euclidien de loi

$$
p=\frac{1}{2}\left[\delta_{r_{\alpha} o \tau}+\delta_{r_{-\alpha} o \tau}\right]
$$


où $r_{\alpha}$ est la rotation $(0, \alpha)$ et $\tau$ la translation horizontale unité. Le résultat d'Eyring est alors conséquence d'un théorème central limite dans le groupe considéré. Dans le problème de localisation la notion de vitesse de fuite, d'exposant de Liapunoff [cf. Infra] joue un rôle essentiel.

Afin de relier brièvement les divers aspects du sujet considéré mentionnons la notion de fonction $p$-harmonique. L'équation de fonction invariante $P f=f$ se traduit par l'équation de convolution : $f * \breve{p}(g)=\int f(g h) d p(h)=f(g)$. Si $f$ est bormée, on peut lui associer la fonction $F \operatorname{sur} \Omega \times G$ définie par

$$
F(\omega, g)=\lim _{n} f\left[g S_{n}(\omega)\right]
$$

car le second membre est une martingale. De plus $f(g)=\int F(g, \omega) d \pi(\omega)$. Il y a correspondance bi-univoque entre les fonctions $p$-harmoniques bornées et les fonctions $\hat{\theta}$-invariantes sur $\Omega \times G$. D'où une interprétation de la notion de fonction harmonique dans le cadre de la théorie ergodique. Par exemple la formule de Poisson dans le disque unité peut être envisagée sous cet angle.

Dans la discussion ci-dessous il est commode de supposer $p$ "adaptée" c'est-àdire que le sous-groupe fermé $G_{p}$ engendré par le support $S_{p}$ de $p$ est égal à $G$. La notion d'"irréductibilité" s'introduit aussi : $p$ irréductible signifie sur le semi-groupe fermé $T_{p}$ engendré par $S_{p}$ est égal à $G$. Enfin $\Omega \pm$ désignera l'espace $G^{\mathbb{Z}}$ et sera muni de la mesure $\pi^{ \pm}=p^{\mathbb{Z}}$ invariante par le décalage bilatère $\theta$.

\section{III - Quelques problèmes fondamentaux.}

Les considérations précédentes, les exemples mentionnés, les connections avec la théorie du potentiel, le calcul des probabilités, la théorie ergodique et l'analyse conduisent à de nombreuses questions de nature générale.

On peut penser que les propriétés asymptotiques de la marche aléatoire sont relativement stables par rapport au choix de la mesure de probabilité $p$ (adaptée), dès que le groupe $G$ est fixé. De ce point de vue on peut donc espérer des relations étroites entre la structure de $G$ et le comportement asymptotique de la marche aléatoire.

Si $G$ est compact, comme dans les exemples 1 et 4 , les propriétés d'équicontinuité des opérateurs $P^{n}$ et l'analyse harmonique de $G$ permettent de préciser le comportement asymptotique de la marche aléatoire par des 
théorèmes généraux d'équirépartition [Gren] et on doit donc distinguer ce cas spécial. Mentionnons quelques-uns des problèmes posés en nous limitant au cas des groupes non compacts.

\section{1) L'étude de l'équation de "Poisson".}

a) L'opérateur de convolution $P-I d$ associé à $p-\delta_{e}$ est l'analogue de l'opérateur de Laplace dans $\mathbb{R}^{d}: \Delta=\sum_{i=1}^{d} \frac{\partial^{2}}{\partial^{2} x_{i}^{2}}$. L'équation de Poisson dans la théorie classique du potentiel est l'équation $\Delta f=-g$; $f$ est le "potentiel" créé par la répartition de charges électriques donnée par g. Dans la situation ici considérée son analogue est donc l'équation intégrale :

$$
(I-P) f=g \text {. }
$$

De manière formelle on est alors conduit à introduire le "noyau potentiel" $V=\sum_{0}^{\infty} P^{n}$ et, dans certaines conditions, $f$ sera donc donnée par

$$
f=\sum_{0}^{\infty} P^{n} g .
$$

Ces considérations posent le problème de la convergence de la série $\sum_{0}^{\infty} P^{n}$, ou plus précisément de la convergence, au sens vague, de la série de puissance de convolution $v=\sum_{0}^{\infty} p^{n}$. On montre que la divergence de cette série équivaut au fait que pour tout $g \geq 0$ (non identiquement nul) l'équation $(I-P) f=g$ n'a pas de solution. Dans la théorie classique du potentiel dans $\mathbb{R}^{d}$, le premier cas a lieu pour $d \geq 3$ et $V$ correspond au potentiel newtonien $V(x, y)=\frac{1}{\|x-y\|^{d-2}}$. Le second cas a lieu pour $d \leq 2$; pour $d=2$, on a la théorie du potentiel "logarithmique" où un analogue non positif de $V$ est le noyau $\log \|x-y\|$.

Dans la situation présente on a la dichotomie

- Pour toute $\varphi \in C_{c}^{+}(G)$, la série $\sum_{0}^{\infty} p^{n}(\varphi)$ converge. C'est le cas dit "transient".

- Pour toute $\varphi \in C_{c}^{+}(G)$, la série $\sum_{0}^{\infty} p^{n}(\varphi)$ diverge. C'est le cas dit "récurrent".

Du point de vue probabiliste, le premier cas correspond au fait que la marche aléatoire $S_{n}(\omega)$ fuit à l'infini dans $G$ et ceci, pour $\pi$-presque tout $\omega$. 
Le second cas correspond au fait que, pour tout voisinage $V$ compact de $e$ et pour $\pi$-presque tout $\omega, S_{n}(\omega)$ effectue une infinité de visites à $V$. On a d'ailleurs l'égalité de $\sum_{0}^{\infty} p^{n}(V)$ et de l'espérance du nombre de visites de $S_{n}(\omega)$ à $V$ :

$$
\sum_{0}^{\infty} p^{n}(V)=\int_{\Omega} \sum_{0}^{\infty} 1_{V}\left[S_{n}(\omega)\right] d \pi(\omega)
$$

Dans la théorie des surfaces de Riemann ouvertes on distingue deux cas [Ts], [Ah] : ou bien la fonction de Green (positive) existe et la surface est dite de type hyperbolique, ou bien la fonction de Green n'existe pas et la surface est dite de type parabolique. Le "problème des types" consiste alors à donner des critères pour qu'une surface ouverte soit de l'un ou l'autre des types.

Le même problème se pose dans le cadre des réseaux électriques [Do-S].

Ici l'analogue de ce problème consiste à donner des critères pour qu'une marche donnée soit récurrente ou transitoire. On l'appellera encore "problème des types". Dans ce cadre il semble avoir été posé pour la première fois par H. Kesten [Ke2].

Dans la situation considérée ici on donne la :

Définition. Un groupe localement compact $G$ est dit récurrent (resp. fortement récurrent) s'il porte une mesure de probabilité adaptée (resp. adaptée et ayant une densité $p$ telle que la série $\sum_{0}^{\infty} p^{n}$ diverge.

Le problème des types se réduit alors à caractériser les groupes récurrents et à préciser la famille des probabilités $p$ telle que la série $\sum_{0}^{\infty} p^{n}$ diverge. On verra plus loin que la forme de la réponse à la première question facilite considérablement la réponse à la deuxième question. Dans le cas récurrent l'expression du noyau potentiel doit être modifiée ; on obtient alors un noyau non positif [Sp], [Bru-Re].

Du point de vue de la théorie ergodique le cas récurrent correspond exactement à l'ergodicité du produit croisé $\Omega \pm \times G$.

b) Si $g$ est donnée et $\mathrm{f}$ satisfait l'équation $(I-P) f=g$, il est clair que $f$ est déterminée modulo les solutions $f^{\prime}$ de l'équation homogène $(I-P) f^{\prime}=0$. Ces solutions sont appelées fonctions $p$-harmoniques par analogie avec la théorie classique du potentiel. Le problème est ici de construire ces fonctions. Plusieurs 
sous-classes de fonctions peuvent être envisagées et jouent un rôle spécial : par exemple les fonctions bornées, les fonctions positives, ou les fonctions "à croissance lente". Le problème le plus simple qui se pose est de caractériser les couples $(G, p)$ tels que les seules fonctions $p$-harmoniques bornées sont les constantes ; plusieurs sous-problèmes peuvent être envisagés. Par analogie avec le théorème de Liouville pour les fonctions holomorphes et la théorie des surfaces de Riemann on donne la définition

Définition. On dit que le couple $(G, p)$ possède la propriété de Liouville si les seules fonctions bornées $f$ qui vérifient l'équation $P f=f$ sont les constantes.

Dans le cas de la théorie du potentiel classique dans $\mathbb{R} d$, les seules harmoniques positives sont les constantes ; la propriété de Liouville est satisfaite. Il n'en est pas ainsi dans le cas du disque unité $D=\{z \in \mathbb{C} ;|z|<1\}$ et du Laplacien $\Delta=\frac{\partial^{2}}{\partial x^{2}}+\frac{\partial^{2}}{\partial y^{2}}$.

En effet le "noyau de Poisson" $P(z, \theta)=\frac{1-|z|^{2}}{\left|e^{i \theta}-z\right|^{2}}=h_{\theta}(z)$ vérifie l'équation $\Delta h_{\theta}=$ 0 et est positif. De plus la formule de Poisson $\left.f(z)=\frac{1}{2 \pi} \int_{0}^{2 \pi} P(z, \theta) \varphi(\theta) d \theta\right)$ où $\varphi$ est continue fournit des fonctions harmoniques non triviales. On connaît les connections étroites entre le disque $D$, ou le demi-plan $H=\{z \in \mathbb{C} ; \operatorname{Im} z>0\}$, et le groupe $G=S \ell(2, \mathbb{R})$ des matrices réelles $(2,2)$ de déterminant 1 : ce groupe agit par transformations conformes sur $D$ et préserve donc les fonctions harmoniques.

Dans le problème de la caractérisation des couples $(G, p)$ possédant la propriété de Liouville, on peut donc s'attendre à des différences essentielles entre des groupes commutatifs tels que $\mathbb{R}^{d}$ et des groupes fortement non commutatifs tels que $S \ell(2, \mathbb{R})$, ou bien le groupe libre à 2 générateurs envisagé en I.

Du point de vue de la théorie ergodique la propriété de Liouville correspond à la constance presque partout des fonctions mesurables bornées sur $\Omega \times G$. Le décalage considéré étant unilatéral et le système de mesure infinie, il s'agit d'une ergodicité au sens faible, contrairement au cas récurrent déjà mentionné.

Lorsque la propriété de Liouville n'est pas satisfaite un des problèmes qui se pose est celui de la représentation intégrale des fonctions $p$-harmoniques bornées par une formule du type de Poisson. L'existence d'une telle 
représentation est assurée par la théorie des algèbres de Banach et la question se réduit donc à expliciter, autant que possible, cette représentation, si le groupe $G$ est défini géométriquement [Fu1], [Ka1], [Ra].

L'exemple qui précède donne lieu également à une description des fonctions harmoniques positives à l'aide d'une formule intégrale $h(z)=\frac{1}{2 \pi} \int_{0}^{2 \pi} h_{\theta}(z) d v(\theta)$ où $v$ est une mesure de Radon positive. Les fonctions $h_{\theta}(z)$ apparaissent alors comme les fonctions élémentaires permettant de construire les fonctions harmoniques générales. Ici encore, pour les fonctions $p$-harmoniques un théorème général de $\mathrm{G}$. Choquet assure l'existence d'une telle représentation intégrale, à l'aide de fonctions "minimales" jouant le rôle des $h_{\theta}(z)$. Le problème qui se pose ici consiste donc à expliciter les fonctions $p$-harmoniques "minimales". On dispose ici d'une méthode générale, due à Martin pour les domaines de $\mathbb{R}^{n}$, mais sa mise en oeuvre exige des informations très précises sur le comportement asymptotique du noyau potentiel $V(x, y)$, informations dont l'obtention est fort délicate et relève, dans le cas des groupes, de l'analyse harmonique $[E \ell],[\mathrm{Bo}$. Les problèmes de comportement au bord des fonctions harmoniques sont également difficiles et ont surtout été abordés pour les Laplaciens [Ko], [Da].

2) Le comportement asymptotique de la position de la marche aléatoire.

Ce comportement présente de nombreux aspects dont la formulation même n'est pas évidente.

Soit $V$ un voisinage compact de l'identité engendrant $G$ [on suppose donc $\left.G=\bigcup_{n>0} V^{n}\right]$. Posons $\delta_{V}(g)=\operatorname{Inf}\left\{n \in N ; g \in V^{n}\right\}$. Dans ce cas où $V$ est un système de générateurs du groupe discret $\Gamma, \delta_{V}(g)$ est la longueur minimum d'une écriture de $g$ comme produit d'éléments de $V$. La fonction $\delta_{V}(g)$ doit être envisagée comme mesurant la "distance" de $g$ à l'origine et le choix de $V$ n'est pas essentiel lorsque $\delta_{V}(g)$ est grand. Une propriété de sous-additivité de $\delta V$

$$
\delta V(g) \leq \delta V(g)+\delta V(h)+\text { Cte }
$$

et le théorème ergodique sous-additif impliquent que $\pi$-presque surement, la limite de $\frac{\delta_{V}\left[S_{n}(\omega)\right]}{n}$ existe et est une constante $\gamma_{V}$ finie si $\int_{G} \delta_{V}(g) d p(g)<+\infty$. 
Définition [Gu2]. Etant donné $V \subset G$ voisinage compact de e, on appelle vitesse de fuite de la marche aléatoire associée à $p$ le nombre défini p.p par

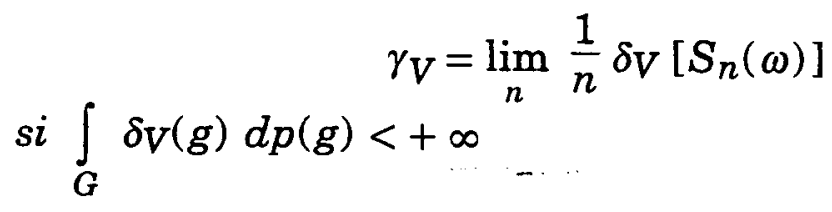

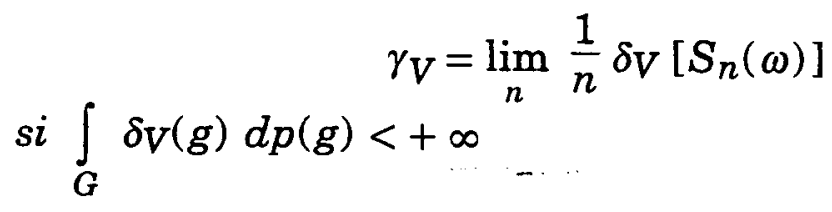

$\mathrm{Si}$ p est fixée, ce nombre dépend de $V$ mais la nullité de $\gamma_{V}$ est indépendante de $V$. Le problème qui se pose ici (dans le cas transient) est donc de caractériser les couples $(G, p)$ tels que $\gamma_{V}>0$.

Il est bien connu que dans le cas d'une marche aléatoire élémentaire symétrique sur $\mathbb{Z}^{d}$ on a $\gamma_{V}=0$. Plus généralement le vecteur $\int_{\mathbb{R}^{d}} x d p(x)$ caractérise, dans $\mathbb{R}^{d}$ la fuite de $S_{n}(\omega)$ et si $\int_{\mathbb{R}^{d}} x d p(x) \neq 0$, on a $\gamma_{V}>\mathbb{R}^{d}$. Dans $\mathbb{R}^{d}$ les deux cas sont donc possibles. On verra que l'on a toujours $\gamma_{V} \neq 0$ dans le cas de $S \ell(2, \mathbb{R})$ ou du groupe libre à 2 générateurs et que cette propriété est reliée à la présence de fonctions $p$-harmoniques bornées non constantes. Cette propriété joue un rôle essentiel dans l'étude de l'exemple 6.

Le comportement directionnel de $S_{n}(\omega)$ est plus difficile à préciser et semble exiger l'introduction d'un "bord à l'infini" de $G$ ou d'une "frontière". On examinera ci-dessous le cas du groupe linéaire où la notion d'exposant de Liapunov s'introduit.

Le problème du comportement en loi de $S_{n}(\omega)$ se pose également [voir l'exemple 3] mais son étude est peu avancée. On peut demander par exemple si la convergence de $\frac{\delta_{V}\left(S_{n}\right)-n \gamma_{V}}{\sqrt{n}}$ vers une loi gaussienne est valide pour tout couple $(G, p)$.

3) Les aspects analytiques du comportement asymptotique des puissances de convolution.

Dans les exemples 1 et 4 , de même que dans le cas général d'un groupe compact on a la convergence de $P^{n} \varphi\left[\varphi \in C_{c}^{+}(G)\right]$ vers $\int_{G} \varphi d m$ et les problèmes qui se posent alors sont plutôt des problèmes de vitesse de convergence. Si $G$ est non compact $P^{n} \varphi$ tend vers zéro et le problème le plus immédiat est celui de 
l'allure asymptotique de $P^{n} \varphi$. Par exemple y-a-t-il convergence exponentielle vers zéro ; peut-on donner un équivalent, ou un encadrement de $P^{n} \varphi(e)$ par des fonctions simples de $n$, par exemple des exponentielles-monomes ?

Ce problème est clairement relié à celui de la convergence de la série $\sum_{0}^{\infty} P^{n}$ mais est de nature beaucoup plus complexe. Dans le cas de $\mathbb{R}^{d}$, une analyse de Fourier précise permet une solution complète, au moins si la probabilité $p$ est à support compact [voir $(\mathrm{Sp})$ pour $\mathbb{Z}^{d}$ ]. Dans le cas général on peut utiliser des méthodes d'analyse harmonique non commutative [Bo] ou un analogue de la théorie de Hardy-Littlewood-Sobolev développée par N.T. Varopoulos [V.S.C]. L'utilisation de telles méthodes exige une connaissance approfondie de la structure de $G$. A un niveau beaucoup plus primitif on peut s'intéresser aux taux de décroissance exponentielle de $P^{n} \varphi$. Deux nombres s'introduisent ici.

Définition. Considérons l'opérateur de convolution sur $\mathbb{L}^{2}(G, m)$ définie par $P \varphi(x)=\left(\varphi * \check{p}(x)=\int \varphi(x g) d p(g)\right.$. On note $r_{0}(p)$ le rayon spectral dans $\mathbb{L}^{2}(G, m)$ de $P$.

Si p est irréductible, on définit

$$
r(p)=\varlimsup_{n}\left[P^{n} \varphi(x)\right]^{1 / n}
$$

où $\varphi \in C_{c}^{+}(G)$. Ce nombre est indépendant du choix de $\varphi$ et de $x$.

On montre aisément que

$$
r(p) \leq r_{0}(p) \leq 1
$$

et que $r(p)=r_{0}(p)$ si $p$ est symétrique. Le nombre $r_{0}(p)^{-1}$ est donc le rayon de convergence de la série d'opérateurs de $\sum_{0}^{\infty} z^{n} P^{n}(z \in \mathbb{C})$ dans $\mathbb{L}^{2}(G, m)$ tandis que le nombre $r(p)^{-1}$ est le rayon de convergence au sens vague de la série de mesures $\sum_{0}^{\infty} z^{n} p^{n}$

Comme ces nombres traduisent la décroissance de la probabilité de présence de $S_{n}(\omega)$ dans un compact de $G$, il est naturel de les comparer à la vitesse de fuite $\gamma_{V}$ définie plus haut. Plus précisément on peut demander quelles sont les relations entre la nullité de $\gamma_{V}$, de $\log r_{0}(p)$ ou $\log r(p)$ et la structure de $G$. 
Par analogie avec le cas des groupes compacts on peut formuler aussi un problème d'équirépartition, si $p$ est irréductible : $\varphi_{0}$ étant un élément fixé de $C_{c}^{+}(G)$, a-t-on convergence vague de la suite de mesures $\frac{p^{n}}{p^{n}\left(\varphi_{0}\right)}$ vers une mesure de Radon?

Sous de larges conditions pour $p$, on peut montrer que si une telle convergence a lieu la mesure limite $\mu$ satisfait l'équation $p * \mu=\mu * p=r(p) \mu$.

Ici la forme précise d'un équivalent de $p^{n}\left(\varphi_{0}\right)$ n'est pas nécessaire à la formulation du problème d'équirépartition. De plus ce problème apparaît lié à l'étude des solutions de l'équation $p * \mu=r(p) \mu$.

On est alors amené à considérer une forme généralisée de l'équation de Poisson de 1) :

$$
(z I-P) f=g \text {. }
$$

Cette équation, comme en 1) conduit à l'étude du $z$-potentiel $\sum_{0}^{\infty} z^{n} p^{n}(z \in \mathbb{C})$.

Sous des conditions assez générales on peut montrer que le rayon de convergence de cette série est égal à $r(p)^{-1}$ et que $p * \mu=r \mu$. a une solution qui est une mesure de Radon positive si et seulement si $r \in[r(p),+\infty[$. Le problème d'équirépartition conduit alors à l'étude de la représentation intégrale des solutions de l'équation $p * \mu=r \mu$ à l'aide de solutions minimales, comme en 1). Cette discussion montre que les divers aspects de l'étude des marches aléatoires sont en fait très reliés. Les méthodes de théorie du potentiel, de calcul des probabilités, de théorie ergodique et d'analyse harmonique mentionnées doivent donc être utilisées de manière complémentaire.

4) Remarques sur certaines classes d'exemples.

Les problèmes mentionnés plus haut sont d'une grande généralité et, pour de nombreuses raisons, il est utile de les étudier dans des cadres plus restreints où l'on peut formuler des réponses plus explicites que dans le cas général. En retour, ces études spéciales conduisent à formuler des conjectures plus générales.

a) Le cas des groupes de Lie connexes.

La structure de $G$ peut être précisée à partir de la donnée de l'algèbre de Lie $\mathscr{G}$, donnant ainsi la possibilité de calculs "d'algèbre linéaire". Le cas des groupes semi-simples présente un intérêt particulier à cause du fait que les structures possibles peuvent être complètement décrites et du fait qu'ils 
interviennent naturellement dans plusieurs questions d'analyse ou de géométrie. De plus, la donnée d'un opérateur hypoelliptique invariant à gauche $\Delta=\sum_{i=1}^{r} X_{i}^{2}+Y\left(X_{i}, Y \in \mathscr{G}\right)$ définit un noyau de convolution dit de la chaleur au moyen de l'équation $\frac{\partial p^{t}}{\partial t}=\Delta p^{t}$.

Le semi-groupe de convolution à temps continu $p^{t}(t>0)$ remplace donc ici le semi-groupe à temps discret $p^{n}(n \in \mathbb{N})$. Par exemple l'équation intégrale $P f=r f=f * \check{p}$ est ici remplacée par l'équation aux dérivées partielles $\Delta f=\lambda f$. Dans ce cas il y a aussi avantage à utiliser des métriques riemanniennes sur $G$. D'autre part, le processus de diffusion associé au semi-groupe $p^{t}$ définit un mouvement brownien $S_{t}(\omega)$ sur $G\left[t \in \mathbb{R}^{+}\right]$qui est l'analogue de la marche aléatoire $S_{n}(\omega)$ considérée plus haut. Des outils nouveaux, en particulier d'analyse peuvent être ici utilisés. Cependant la nature des problèmes asymptotiques considérés ici $(t \rightarrow+\infty)$ fait que l'on ne peut s'attendre à des résultats essentiellement plus simples que dans le cas général d'une mesure de probabilité ayant une densité.

b) Le cas des groupes discrets.

Les intégrales sont ici remplacées par des séries convergentes ou même des sommes finies. Des méthodes spéciales comme celles de la théorie des graphes peuvent être utilisées. C'est en particulier le cas si $p$ est à support fini et symétrique car, dans ce cas, les problèmes admettent une interprétation en termes de réseaux électriques, ce qui permet d'utiliser des méthodes connues en électricité. Enfin les groupes discrets sont souvent définis dans un contexte géométrique qui lui aussi permet des interprétations géométriques et l'utilisation du calcul différentiel sur les variétés.

c) Le cas des groupes de points rationnels d'un groupe algébrique défini sur un corps local $\mathbb{F}$.

Le groupe $G$ des points $\mathbb{F}$-rationnels est localement compact et si $\mathbb{F}$ est ultramétrique ou dispose de techniques de réduction au cas discret ou même fini, ce qui compense partiellement l'absence de calcul différentiel. On peut donc s'attendre à des résultats analogues au cas des groupes de Lie, les méthodes prenant ici un aspect combinatoire.

d) Le cas des probabilités ayant une densité par rapport à la mesure de Haar.

Dans ce cas la mesure $p$ s'identifie à une fonction et les méthodes d'analyse fonctionnelle sont très bien adaptées. De plus, dans ce cas le rôle de la 
structure du groupe $G$ est prédominant par rapport à celui de la mesure. A contrario le cas des mesures étrangères par rapport à la mesure de Haar, atomiques par exemple, est susceptible de faire intervenir des propriétés du support de la mesure de nature arithmétique. Par exemple les problèmes d'équirépartition deviennent particulièrement intéressants et difficiles : les situations correspondantes sont proches de celles de la théorie ergodique. Enfin si la probabilité $p$ est symétrique l'opérateur qu'elle définit sur $\mathbb{L}^{2}(G, m)$ est auto-adjoint et cette particularité permet l'application des méthodes d'espaces de Dirichlet, d'énergie, de théorie spectrale. L'analogie avec la théorie de l'électricité prend ici toute sa valeur.

\section{IV - Quelques résultats.}

Nous illustrons ici quelques-uns des sujets évoqués en III en décrivant les résultats, l'interaction des méthodes utilisées et le développement historique correspondant :

1) Le problème des types et la structure des groupes récurrents.

Dans le cas des groupes abéliens engendrés par un compact $V$, une étude assez complète de ce type de problème peut être menée à l'aide de l'analyse de Fourier : les seuls groupes récurrents sont $\{0\}, \mathbb{Z}, \mathbb{Z}^{2}, \mathbb{R}, \mathbb{R}^{2}, \mathbb{R} \times \mathbb{Z}$ et leurs produits par des groupes compacts [cf Sp].

Dans le cas des groupes discrets non commutatifs finiment engendrés il a été conjecturé par $\mathrm{H}$. Kesten [Ke2] que les groupes récurrents sont à "croissance non exponentielle". Nous devons ici préciser quelques définitions et résultats préliminaires.

Définition. Soit $G=\cup_{n>0} V^{n}$ un groupe localement compact engendré par le voisinage compact de l'identité $V$ et muni d'une mesure de Haar (à droite) $m$. On dit que $G$ est à croissance polynomiale de degré $\leq d$ (resp. exponentielle) s'il existe une constante $C$ telle que

$$
m\left(V^{n}\right) \leq C n^{d}
$$

$\left[\operatorname{resp} \lim _{n}\left[m\left(V^{n}\right)\right]^{1 / n}>1\right]$

Remarque. On a toujours $\underset{n}{\lim }\left[m\left(V^{n}\right)\right]^{1 / n} \geq 1$ et $\mathrm{R}$. Grigortchuk [Gri] a donné de nombreux exemples de groupes discrets qui ne sont pas à croissance 
polynomiale tels que $\lim \left[m\left(V^{n}\right)\right]^{1 / n}=1$ [Gri]. Les groupes abéliens, nilpotents ou de type rigide sont à croissance polynomiale [Gu1].

Un résultat fondamental de $M$. Gromov (Gro) décrit la structure des groupes finiment engendrés et à croissance polynomiale, répondant ainsi à une conjecture de J. Milnor [Mi].

Théorème [Gro]. Soit $\Gamma$ un groupe de type fini à croissance polynomiale. Alors $\Gamma$ contient un sous-groupe nilpotent d'indice fini.

Remarque. Dans le cas général d'un groupe localement compact il est possible de décrire la structure des groupes à croissance polynomiale [Lo] en utilisant une classe de groupes introduite en [Gu1] et qui généralise les groupes de type rigide.

Définition [Gree] [Pi]. Un groupe localement compact est dit moyennable s'il existe sur $\mathbb{L} \infty(G, m)$ une forme linéaire $\lambda$ positive invariante par translation et telle que $\lambda(1)=1$.

Remarque. Les groupes à croissance non exponentielle sont moyennables mais il existe des groupes moyennables à croissance exponentielle dont en particulier certains groupes résolubles, comme le groupe affine de la droite.

La conjecture de $\mathrm{H}$. Kesten était justifiée par une étude détaillée des groupes polycycliques et par le fait que pour un groupe discret non moyennable on a toujours $r(p) \leq r_{0}(p)<1$, donc la transience [Ke1].

A cause des relations étroites entre groupes discrets et groupes de Lie il était naturel d'étudier l'analogue de la conjecture de Kesten dans le cadre des groupes de Lie et de leurs sous-groupes. Dans le travail [Gu-K-R] la conjecture de Kesten a été précisée et généralisée de la façon suivante.

Conjecture [Gu-K-R]. Un groupe localement compact à génération compacte est récurrent si et seulement s'il est à croissance polynomiale de degré 2 au plus.

Il ne semble pas que cette conjecture soit actuellement établie en toute généralité. Elle a cependant été établie sous des hypothèses complémentaires par N.T. Varopoulos. Les méthodes de [V.S.C] permettent d'obtenir l'énoncé suivant :

Théorème. Soit Gun groupe discret finiment engendré. Alors si $G$ est récurrent, c'est une extension finie de $\mathbb{Z}$ ou $\mathbb{Z}^{2}$. 
La méthode utilise essentiellement le théorème de M. Gromov déjà cité , les espaces de Dirichlet, ainsi qu'une extension de la théorie de Hardy-LittlewoodSobolev où les inégalités isopérimétriques jouent un rôle important. Ce théorème résout donc en particulier la conjecture de $H$. Kesten sous sa forme originale et fournit également la structure des groupes récurrents. Ces méthodes généralisées au cas des groupes localement compacts ne permettent pas apparemment de résoudre le cas général. Une difficulté importante réside dans l'étude des probabilités adaptées mais ne possèdant pas de densité par rapport à la mesure de Haar. Grâce aux travaux [Gu1], [Lo] mentionnés plus haut et aux méthodes de [V-S-C], on peut obtenir le résultat suivant :

Théorème. Si $G$ est à génération compacte il est fortement récurrent si et seulement s'il est égal à $\mathbb{Z}$ ou $\mathbb{Z}^{2}$ à des extensions compactes près.

La difficulté liée aux mesures singulières a été résolue dès les années 70 si le groupe $G$ admet une injection continue dans un groupe de Lie connexe.

Théorème (Gu-K-R). Soit Gun groupe localement compact à génération compacte et admettant un homomorphisme injectif dans un groupe de Lie connexe. Alors $G$ est récurrent si et seulement s'il possède un sous-groupe compact distingué $C$ tel que $G / C$ soit extention compacte de $\mathbb{Z}$ ou $\mathbb{Z}^{2}$. En particulier si $G$ est un groupe de Lie connexe et non compact, il est récurrent si et seulement si il est extension compacte de $\mathbb{R}$ ou $\mathbb{R}^{2}$.

Ce théorème ne comporte donc pas d'hypothèse sur la nature des mesures de probabilités. La théorie des groupes de Lie est un outil important qui permet de résoudre cette difficulté. La méthode consiste à se ramener à certains groupes de basses dimensions où une étude détaillée est possible. Cette réduction, par passage au quotient, est possible à cause du lemme suivant qui se révèle très efficace.

Lemme. Si G est récurrent il est moyennable et unimodulaire.

Les preuves peuvent être simplifiées à l'aide d'un résultat de [Ba-L-P] qui permet de se ramener au cas des probabilités symétriques et à support compact. Ce résultat paraît être le premier exemple d'utilisation des méthodes d'espaces de Dirichlet dans ce problème.

On voit donc que la structure simple des groupes récurrents obtenus doit permettre une étude détaillée des critères de récurrence pour une mesure de probabilité $p$. 
Un exemple typique est le groupe des déplacements euclidiens de $\mathbb{R}^{2}$, qui a joué un rôle important dans l'étude des conjectures précédentes. Dans ce cas l'existence du moment d'ordre 2 implique la récurrence [Cr]. Dans le cas des groupes $\mathbb{Z}^{d}$ on dispose d'une condition nécessaire et suffisante de transience en termes de transformée de Fourier :

$$
\int_{\mathbb{T}^{d}} \operatorname{Re} \frac{1}{1-\hat{p}(\theta)} d \theta<+\infty
$$

où $\hat{p}(\theta))=\sum_{x \in Z^{d}} p(x) e^{i<x, \theta>}$ et seul le cas $d \leq 2$ est intéressant [Sp]. Une étude détaillée des groupes de croissance au plus 2 est susceptible de mener à un critère analogue.

L'étude du problème posé en [Ke2] et [Gu-K-R] a donc mis en jeu des méthodes variées de théorie du potentiel de calcul des probabilités, d'analyse et de théorie des groupes. L'interprétation en termes de réseaux électriques [N-W], [Ly] a elle aussi joué un rôle important en mettant en évidence des méthodes indépendantes de l'analyse de Fourier qui ont été efficacement mises en oeuvre en [V.S.C] dans le contexte plus général de l'Analyse sur les groupes de Lie.

\section{2) La propriété de Liouville.}

Les solutions de l'équation de Poisson $(I-P) f=g$ sont déterminées à une fonction harmonique près, et dans le cas transient si $g$ est continue à support compact, l'une de ces solutions est le potentiel Vg. De plus le principe du maximum montre que $V$ est "borné par translation", c'est-à-dire que pour tout voisinage compact $U$ de $e$ dans $G$ on a

$$
\sup _{x, y \in G} V(x U y)<+\infty
$$

Les solutions bornées de l'équation de Poisson seront donc définies à une constante près par la relation $f=V g$ dès que le groupe $(G, p)$ possède la propriété de Liouville. Dans le cadre de la variable complexe et des fonctions holomorphes, la propriété correspondante est la constance des fonctions holomorphes bornées. On connaît l'importance de cette propriété dans le cadre de l'étude des surfaces de Riemann. On peut se demander à quelles conditions sur $(G, p)$ la propriété de Liouville est satisfaite. L'étude de ce problème est loin d'être achevée et il est commode de décrire les résultats connus à partir de la situation la plus étudiée, celle où la probabilité $p$ admet une densité $p(g)$ par rapport à une mesure de Haar à droite fixée. 
Définition (Av). On appelle entropie du couple $(G, p)$ le nombre $h(G, p)=\overline{\lim }-\frac{1}{n} \int p^{n}(g) \log p^{n}(g) d g$ où $p^{n}(g)$ est la densité de la $n^{i e ̀ m e ~}$ puissance de convolution de $p$.

Remarques. a) Ce nombre est fini dès que $p(g)$ est continue à support compact. Il ne dépend pas du choix de la mesure de Haar droite mais n'est pas invariant par symétrie en général.

b) On voit aisément que $h(G, p)=0$ si $G$ est à croissance non exponentielle ou bien si la vitesse de fuite est nulle. Inversement $\operatorname{si}(G, p)=0, G$ est moyennable (Gu2). La nullité de $h$ met en jeu les propriétés de $G$ et celles de $p$.

c) Dans le cas d'une probabilité symétrique à support compact et d'une groupe unimodulaire la nullité de $h$ équivaut à celle de la vitesse de fuite (V2).

Théorème (De, Ka-V). Supposons $h(G, p)<+\infty$. On a $h(G, p)=0$ si et seulement le couple $(G, p)$ a la propriété de Liouville.

Dans le cas des groupes de Lie connexes on trouvera des résultats plus précis en $[\mathrm{Bi}-\mathrm{R}]$, en termes de vitesse de fuite. En particulier on a le

Théorème 5 (Az). Soit $G$ un groupe de Lie connexe à croissance polynomiale. Alors pour toute probabilité ayant une densité, le couple $(G, p)$ a la propriété de Liouville.

Remarques. a) Dans [Az] ce résultat est formulé en termes de la structure de $G$. $G$ est dit alors de type rigide ; les groupes de déplacements euclidiens et les groupes nilpotents sont de type rigide. La preuve repose sur des arguments de martingales qui ont été très développés ensuite par A. Raugi [Ra].

b) Ce résultat a été retrouvé en [Gu1] par des méthodes d'Analyse harmonique et la notion de croissance y a été mise en évidence. Ces méthodes permettent aussi l'étude du cas où $p$ est singulière par rapport à la mesure de Haar [cf. Infra].

c) Une partie de ces résultats a été retrouvée en [Av], et a justifié l'introduction de la notion d'entropie ; ceci fournit l'une des implications du théorème 4. L'autre implication repose sur une formule générale donnant $h(G, p)$ en termes de l'espace de Poisson de $(G, p)$, formule qui apparaît déjà en [Fu3]. Le rôle de la moyennabilité dans le problème considéré est décrit par le 
Théorème 3 (Ro). Le groupe G est moyennable si et seulement s'il existe une probabilité $p$ à densité continue telle que $(G, p)$ ait la propriété de Liouville.

Remarques. a) Dans le cas $G$ de Lie connexe, ce théorème avait été obtenu en [Bi-Ra] en utilisant la structure de $G$.

b) Si $p$ est singulière par rapport à la mesure de Haar mais adaptée, la propriété de Liouville pour $(G, p)$ implique la moyennabilité de $G$, ce qui avait été montré antérieurement en [Gu1].

Tenant compte de ces résultats on peut formuler la

Conjecture. Le groupe $G$ est à croissance non exponentielle si et seulement si pour toute probabilité à densité continue et à support compact, le couple $(G, p)$ possède la propriété de Liouville.

Dans le cas où $p$ est singulière mais adaptée, on ne dispose que de résultats fragmentaires. La conjecture suivante a été formulée en [Fu4].

Conjecture [Fu4]. Si $G$ est nilpotent et $p$ adaptée, le couple $(G, p)$ possède la propriété de Liouville.

Le théorème suivant [Gu1] résout cette conjecture sous des hypothèses de moment ou de densité additionnelles [voir III 2].

Théorème [Gu1]. Supposons G nilpotent et $p$ adaptée. S'il existe $\varepsilon>0$ tel que $\int \delta_{V}^{\varepsilon}(g, d p(g)<+\infty$ ou bien si $G$ est de classe deux au plus alors $(G, p)$ possède la propriété de Liouville. Cette propriété est valide aussi si $p$ ou l'une de ses puissances de convolution est non singulière par rapport à la mesure de Haar.

Remarques. a) La deuxième partie découle aussi des méthodes développées en [Az]. La première partie utilise des arguments de représentations unitaires et des arguments de croissance.

b) La propriété de Liouville est aussi valide si $G$ est le produit semi-direct d'un groupe compact $C$ abélien et d'un groupe nilpotent $N$ et $p$ satisfait une condition de moment [Gu1].

Par contre si $C$ n'est pas abélien, la validité de la propriété de Liouville ne semble pas connue. Le cas le plus élémentaire de ce type est celui du groupe des déplacements de l'espace euclidien de dimension $\geq 3$ et d'une probabilité atomique. 
Cette situation peut être comparée à celle concernant les théorèmes quotients et les théorèmes locaux et les problèmes d'équirépartition [voir III 3)].

c) Si $G$ est abélien, la validité de la propriété de Liouville est un fait bien connu dû à G. Choquet et J. Deny [Ch-D] et d'usage essentiel dans la théorie du renouvellement $[\mathrm{F}]$. Ce résultat découle aussi des propriétés de synthèse spectrale pour les groupes abéliens [Gu1].

3) La simplicité du spectre de Liapunov.

On a mentionné en III 2) les problèmes de vitesse de fuite de la marche aléatoire. Dans cette direction un résultat frappant a été obtenu par H. Furstenberg au début des années 60 [Fu2].

Théorème. Soit $p$ une probabilité sur le groupe linéaire $S L(d, \mathbb{R})$ et $S_{n}(\omega)$ le produit de matrices aléatoires $S_{n}(\omega)=A_{n}(\omega)$... $A_{1}(\omega)$. Supposons que $G_{p}$ soit non compact et ne laisse invariante aucune réunion finie de sous-espaces vectoriels. Alors si $\int \log \|g\| d p(g)<+\infty$, il existe une constante strictement positive $\gamma>0$ telle que l'on ait, presque partout

$$
\lim _{n} \frac{1}{n} \log \left\|S_{n}\right\|=\gamma \leq \int \log \|g\| d p(g) .
$$

Remarques. a) Ce type de résultat, qui peut être considéré comme une loi des grands nombres a été précisé par une série de théorèmes limites dont le théorème central limite, sous des hypothèses additionnelles [Tu1], [Vi].

b) Pour le mouvement brownien hyperbolique du disque unité, ce théorème dit que chaque trajectoire converge vers un point déterminé du cercle unité, avec une vitesse non nulle.

c) Dans les exemples l'estimation de la constante $\gamma$, qui est strictement positive, est un problème difficile. La preuve ne fournit pas de formule simple pour $\gamma$.

d) Une forme abstraite de ce résultat, utile en géométrie, qui en retient les aspects essentiels a été donnée en [Gu2], donnant ainsi l'énoncé suivant.

Théorème. Soit $p$ une probabilité adaptée sur un groupe localement compact. G engendré par un compact $V$. Alors si $G$ est non moyennable et si $\int \delta V(g) d p(g)$ $<+\infty$, il existe un nombre $\gamma_{V}>0$ tel que l'on ait p.p:

$$
\lim _{n} \frac{\delta V\left(S_{n}\right)}{n}=\gamma_{V}
$$


Ces théorèmes ne tiennent pas compte des aspects "directionnels" du comportement de $S_{n}(w)$ et donnent donc souvent une information très partielle par exemple dans le cas des groupes linéaires $G=S \ell(d, \mathbb{R}) \quad(d>2)$.

On va préciser certains aspects directionnels dans ce cas particulier. D'autres cas présentent un intérêt physique comme celui du groupe symplectique $S p(2 n, \mathbb{R})$ qui intervient par exemple pour l'étude de la propagation des ondes dans les guides d'ondes présentant des inhomogénéités [Tu2]. Notons $K=S O(d)$ le groupe des rotations, $A$ le groupe des matrices diagonales à coefficients positifs, $A^{+}$le sous-ensemble des matrices $a=\operatorname{diag}\left(a_{1}, a_{2}, \ldots, a_{d}\right)$ avec $a_{1} \geq a_{2} \geq \ldots \geq a_{d}$. On sait que toute matrice inversible $g$ peut se décomposer sous forme polaire $g=k a k^{\prime}$ avec $k, k^{\prime} \in K, a \in A^{+}$et $a$ est défini de manière unique. Alors le théorème ergodique multiplicatif $[\mathrm{O}]$ permet de poser la

Définition. On suppose que $\int \log \|g\| d p(g)<+\infty$ et $\int \log \left\|g^{-1}\right\| d p(g)<+\infty$. On appelle spectre de Liapunov du produit de matrices aléatoires $S_{n}(\omega)=g_{n} \ldots$ $g_{1}=K_{n} A_{n} K_{n}^{\prime}$ la matrice diagonale $\gamma=\operatorname{diag}\left(\gamma_{1}, \gamma_{2}, \ldots, \gamma_{d}\right)\left(\gamma_{1} \geq \gamma_{2}, \ldots, \geq \gamma_{d}\right)$ définie par $\gamma=\lim _{n} \frac{\log A_{n}}{n}$.

Les nombres $\gamma_{i}$ sont appelés les exposants de Liapunov de $S_{n}(\omega)$.

Remarques. a) L'existence ds $\gamma_{i}$ ne dépend pas de l'indépendance des $g_{k}(w)$ mais de leur stationnarité.

b) Les $\gamma_{i}$ sont les analogues des logarithmes des valeurs absolues des valeurs propres d'une matrice $g \in G \ell(d, \mathbb{R})$. Par exemple $\gamma_{1}$ correspond au logarithme du rayon spectral de $g$.

c) Dans les exemples, l'estimation des $\gamma_{i}$ est un problème difficile.

Dans les applications, on est en particulier intéressé par des inégalités entre les $\gamma_{i}$. On dit que le spectre de Liapunov est simple si $\gamma_{1}>\gamma_{2}>\ldots>\gamma_{d}$.

d) En [Fu2] il a été conjecturé que si $d>1$ et si $p$ a une densité sur $G \ell(d, \mathbb{R})$ alors le spectre de Liapunov est simple. Sous cette forme la conjecture a été établie par V. Tutubalin [Tu2] en se basant sur le théorème central limite puis par A. Raugi [Ra], A. Virtser [Vi] en utilisant des méthodes plus directes. Le cas des probabilités singulières se présente naturellement dans les applications aux milieux désordonnés. Par exemple si $A$ et $B$ sont deux matrices en position suffisamment générale, $p=\frac{1}{2}\left(\delta_{A}+\delta_{B}\right)$ est un cas typique. 
e) La simplicité du spectre de Liapunov a été remarquée pour la première fois, semble-t-il, par E.B. Dynkin dans le cadre de l'étude de la compactification de Martin pour le mouvement brownien sur l'espace symétrique des matrices hermitiennes définies positives de déterminant 1 [Dy]. Par la suite, ce phénomène a fait l'objet d'études approfondies par de nombreux auteurs. Des idées importantes ont été dégagées par $\mathrm{H}$. Furstenberg [Fu4] et formulées en termes de dynamique topologique.

Afin de formuler un résultat essentiel notons $\wedge^{k} \mathbb{R}^{d}(1 \leq k \leq d)$ l'espace des $k$ multivecteurs. On rappelle que l'action de $g \in G \ell(d, \mathbb{R})$ sur $\wedge^{k} \mathbb{R}^{d}$ est donnée $\operatorname{par} g\left(v_{1} \wedge v_{2} \wedge \ldots \wedge v_{k}\right)=g v_{1} \wedge g v_{2} \wedge \ldots \wedge g v_{k}$.

Théorème (Gu3, Gu-R1). Soit $T_{p}$ le semi-groupe fermé de $G \ell(d, \mathbb{R})$ engendré par le support de $p$. On suppose que Tp contient une matrice diagonalisable à valeurs propres distinctes en module et que l'action de Tp sur $\wedge^{k} \mathbb{R}^{d}(1 \leq k<d)$ ne laisse invariante aucune réunion finie de sous-espaces vectoriels. Alors le spectre de Liapunov du produit de matrices aléatoires associé est simple.

Remarques. a) Ce théorème a été amélioré par I. Goldcheid, G. A. Margulis [Go-M] qui ont montré que les hypothèses du théorème étaient satisfaites dès que $T_{p}$ était dense au sens de Zariski dans $G \ell(d, \mathbb{R})$ ou $S \ell(d, \mathbb{R})$. Ces hypothèses sont donc très peu contraignantes et sont satisfaites dans les cas typiques $p=\frac{1}{2}\left(\delta_{A}+\delta_{B}\right)$. Cette condition de densité est souvent aisément vérifiable dans les applications.

b) La preuve du théorème esquissée en [Gu3] est non constructive et repose sur des arguments de théorie ergodique et de convergence de certaines martingales.

c) Ce théorème donne accès à de nombreux résultats de calcul des probabilités, beaucoup plus précis, en utilisant des arguments de théorie spectrale des opérateurs. On peut ainsi obtenir sous de très larges conditions des théorèmes limites centraux, des théorèmes locaux, de renouvellement, des grandes déviations, etc... [voir (Ke3), (Bo-L), (LeP), [Go-G], [Gu-R2], (Let)].

\section{V - Remarques sur le développement historique.}

En II ont été motivées les grandes lignes de développement du sujet présenté. Le cas des groupe abéliens a fait l'objet d'études nombreuses et approfondies en combinant l'analyse de Fourier et la théorie du potentiel ; d'un point de vue probabiliste c'est une extension de l'étude des sommes de variables 
aléatoires indépendantes. On se borne à renvoyer à l'ouvrage de F. Spitzer [Sp] qui concerne le cas de $\mathbb{Z}^{d}$. On peut trouver dans l'ouvrage de U. Grenander [Gren] plusieurs résultats connus au début des années soixante qui concernent certains groupes non abéliens. Aux environs des années soixante les aspects probabilistes se sont d'abord développés, motivés par l'étude des relations de récurrence à coefficients aléatoires, avec les travaux de $H$. Kesten, H. Furstenberg, V. Tutubalin. Les aspects de théorie du potentiel ont ensuite joué un rôle important avec par exemple les travaux de H. Kesten, A. Brunel, D. Revuz, R. Azencott, Y. Guivarc'h, A. Avez, Y. Derriennic, A. Raugi, P. Bougerol, P. Crépel, L. Élie. On pourra se reporter à l'ouvrage [Gu-K-R] pour la partie de ces travaux concernant le problème des types et aux travaux [Gu2], [Gu5], [Ra] pour un exposé des résultats connus avant les années quatre vingt dans le domaine des fonctions harmoniques et des relations de récurrence à coefficients aléatoires, à [Gu6] pour un exposé d'ensemble plus récent.

On peut noter dans cette période une forte interaction entre calcul des probabilités, théorie du potentiel et théorie des groupes. Les aspects de théorie ergodique de ces problèmes ont d'abord été développés par H. Furstenberg [Fu4] autour des années soixante dix, puis par $\mathrm{R}$. Zimmer [ $\mathrm{Zi}$ ] en relation avec les problèmes de rigidité des réseaux des groupes de Lie. Ces aspects ont été développés dès le début des années quatre vingt par Y. Guivarc'h, F. Le Drappier, A. Raugi, E. Le Page, V. Kaimanovich, M. Babillot, A. Ancona, en particulier en relation avec des problèmes de nature géométrique ou dans le cadre de l'étude des exposants de Liapunov. On pourra trouver une partie de ces développements dans l'ouvrage de P. Bougerol et J. Lacroix (Bo-L).

Les aspects géométriques ne sont pas abordés dans ce dernier ouvrage et débordent d'autre part du cadre de théorie des groupes donné en II. Ils concernent aussi bien les produits de matrices aléatoires et les systèmes dynamiques hyperboliques que la théorie du potentiel sur les variétés ou les théorèmes ergodiques non commutatifs. On pourra consulter les articles [Gu$\mathrm{H}$ ], [Gu4], [Br-D-P] pour le premier aspect, les travaux [ Fu3], [An], [Ba], [LeD], [Ly-S], [Li-P], [Ka2] et [Gu-J-T) pour le second aspect, et enfin le travail [Ne] pour le dernier aspect.

A peu près à la même époque s'est développée sous l'impulsion de T. Lyons [Ly], N.T. Varopoulos [V3], [Co-S] une autre direction se rattachant aux "méthodes hilbertiennes". Ces idées permettent en faisant jouer un rôle essentiel à la réversibilité, de résoudre une partie des questions laissées ouvertes dans les années soixante dix, concernant en particulier le problème des types et 
l'asymptotique du noyau de la chaleur sur les groupes de Lie. On pourra se reporter à l'ouvrage [V.S.C.] et aux articles [He-S], [V1], [Co-S] pour des exposés de ces résultats, basée sur des méthodes difficiles d'Analyse. Enfin l'aspect "théorie des graphes" est d'un développement plus récent et peut être considéré comme un prolongement de cette direction. Les propriétés spectrales des opérateurs de transition y jouent un rôle important ainsi que les inégalités isopérimétriques. On pourra se reporter aux articles de synthèse [Wo], [Gri$\mathrm{DlH}$ ]. Pour un aperçu de ces travaux on pourra consulter par exemple les travaux [Car], [Be-P], [Co--S], [Wo], [So] et les références qui y sont indiquées. L'article [Wo] contient une description de ces travaux, de nombreuses références et on y renvoie pour plus de détails.

Il contient aussi une description de quelques aspects des travaux relatifs aux marches aléatoires sur les graphes et les groupes discrets. Pour un exposé de ces questions sous l'angle de la théorie du potentiel discret sur les graphes, on pourra consulter l'ouvrage [So]. En ce qui concerne les marches aléatoires sur les groupes (non abéliens) on pourra se reporter pour des exposés synthétiques mais limités à des aspects choisis, aux ouvrages [Gu-K-R], [Bo-L], [V-S-C], [GuJ-T] ainsi qu'aux articles [Ke2], [Fu3], [Fu4], [Gu2], [Gu5], [Ka-V], [Ka2], [Gu6], [V1], [Gri-Dlh]. On peut donc observer une grande diversification de ce sujet depuis les années soixante dix, tant du point de vue des méthodes, que de celui des questions abordées. Les aspects probabilistes restent très importants mais des apports très substanciels ont eu lieu aussi bien du point de vue de la théorie ergodique et de la géométrie, que des applications physiques, des méthodes analytiques ou de théorie des graphes. Un double travail s'effectue : l'amélioration des résultats existants qui tendent à prendre une forme définitive et la résolution de questions particulières nouvelles suggérées par d'autres domaines. Il semble que les progrès les plus importants ont eu lieu dans les problèmes relatifs aux fonctions harmoniques, le problème des types, les théorèmes limites de caractère probabiliste, l'asymptotique du noyau de la chaleur, les problèmes de frontières et de compactifications, les inégalités isopérimétriques et les propriétés spectrales. Cependant de nombreuses questions ne sont que très partiellement résolues. Par exemple, l'étude des fonctions harmoniques bornées, des fonctions harmoniques positives et des compactifications reste très incomplète (voir [Gu-J-T], [Ka2]. Il en est de même des questions relatives aux théorème central limite et aux asymptotiques gaussiennes (voir [Bo-L], [V1], [He-S]). Les relations entre propriétés spectrales, 
entropie, vitesse de fuite, croissance, fonctions harmoniques demandent à être précisées ([Gri-Dlh], [Gu6]).

La bibliographie suivante tente d'éclairer les principaux développements du sujet dans le cadre de théorie des groupes quelque peu réducteur développé cidessus. Cette bibliographie est très incomplète ; elle comporte des articles importants par leur nouveauté, des ouvrages ou des articles de synthèse.

\section{Bibliographie}

[A-K] V.I. ARNOLD, A.L. KRYLOV - Uniform distribution of points on a sphere and some ergodic properties of solutions of differential equations in a complex region, Sov. Math Dokl $4 \mathrm{n}^{\circ} 1, \mathrm{p} 1-5,1963$.

(Ah) ALHFORS - Conformal invariants. Topics in geometric function theory, Mac Graw Hill, New York, 1973.

(An) A. ANCONA - Théorie du potentiel sur les graphes et les variétés, Lecture Notes in Math. 1427, p 3-112, 1998.

(Av) A. AVEz - Théorème de Choquet-Deny pour les groupes à croissance non exponentielle, C.R.A.S. 279, p 25-28, 1974.

(Az) R. AzEnCOTT - Espaces de Poisson des groupes localement compacts, Lecture Notes in Math. 148 Springer, 1970.

(B) BANACH - Sur le problème de la mesure, Fund Math 4, p 7-33, 1923.

(Be-P) BENJAMINI-PÉRÈS - Random walk on a tree and capacity in the interval, Ann. I.H.P., 1991.

(Ba-L-P) P. BALdi, N. LOHOUÉ, J. PEYRIÈRE - Sur la classification des groupes récurrents, C.R.A.S. t. 285, p 1103-1104, 1977.

(Ba) M. BABILLOT - Théorie du renouvellement pour les chaînes semimarkoviennes transientes, Ann. I.H.P., vol. 24, nº 4, p 507-569, 1998.

(Bo-L) P. BOUGEROL, J. LACROIX - Products of random matrices with applications to Schroëdinger operators, Progress in Probability and Statistics 8, Birkhaüser, 1985.

(Bi-R) BIRGÉ, A. RAUGI - Fonctions harmoniques sur les groupes moyennables, C.R.A.S. 278, p 1287-1289, 1974.

(Bo) P. Bougerol - Comportement à l'infini du noyau potentiel du mouvement brownien sur un espace de Riemannien symétrique, Lecture Notes in Math. 1096, p 90-115, 1983. 
(Br-D-P) A. BROISE, F. DAL'BO, M. PEIGNÉ - Études spectrales d'opérateurs de transfert et applications, Astérisque 238, 1998.

(Bru-Re) A. BRUNEL, A. REVUZ - Marches de Harris sur les groupes localement compacts, Ann. E.N.S., (4), 7, p 273-310, 1974.

(C) CARTAN - Théorie générale du balayage en potentiel newtonien, Ann. Univ. Grenoble Math. Phys. 22, p 221-280, 1946.

(Ca-L) R. CARMONA, J. LACROIX - Spectral theory of random Schroëdinger operators, Probability and its applications Birkhaüser, 1990.

(Car) CARTWRIGHT - On the asymptotic behaviour of convolution powers of probabilities on discrete groups, Monatsh Math. 107, p 287-290, 1989.

(Ch-D) G. CHOQUET, J. DENY - Sur l'équation de convolution $\mu=\mu * \sigma$, C.R.A.S. t. 250, p 799-801, 1960.

(Co-S) T. CoUlhoN, L. SALOFF-CosTE -Isoperimetrie pour les groupes et les variétés, Rev. Mat. Iberoamericana 9, p 293-314, 1993.

(Cr) P. CRÉPEl - Récurrence des marches aléatoires sur les groupes de Lie, Lecture Notes in Math. 532, p 50-69, Springer, 1973.

(D) DENY - Méthodes hilbertiennes en théorie du potentiel, C.I.M.E. Rome ed. Cremonese, 1970.

(Da) DAMEK - Pointwise estimates for the Poisson kernel on AN-groups by the Ancona method, Annales de la Faculté des Sciences de Toulouse, à paraître, 1998.

(De) Y. DERRIENNIC - Frontière de Martin pour les marches aléatoires sur le groupe libre, Z. Wahr 32, p 261-276, 1975.

(Di) DIACONIS - Applications of non commutative Fourier analysis to probability problems, Lecture Notes in Math. 1362, p 51-100, 1988.

(Do-S) DOYLE-SNELL - Random walks and electrical networks, Carus Math. Monographs 22, 1988.

(Dy-M) E.B. DYNKIN - M. MALYUTOV - Random walks on groups with a finite number of generators, Soviet Math. Dokl 2, p 399-402, 1961.

(Dy) E.B. DYNKIN - Non negative eigenfunctions of the Laplace-Beltrami operator in certain symmetric spaces, Math. USSR Isvestyia 72, p 203-226, 1968.

(Eck) ECKMANN - Lois de Kirchoff et fonctions discrètes harmoniques, Bull. Soc. Vaud Sc Nat 63, n² 261, Lausanne, 1945.

(El) L. ELIE - Comportement asymptotique du noyau potentiel sur les groupes de Lie, Ann. E.N.S. t. 15, p 257-364, 1982. 
(F) FELLER - An introduction to probability theory and its applications, vol. I, II, $3^{\mathrm{d}}$ ed., New York, 1968.

(Fu1) H. Furstenberg - A Poisson formula for semi-simple Lie groups, Annals of Math. 2, t. 77, p 335-386, 1963.

(Fu2) H. FURSTENBERG - Non commuting random products, TAM.S 108, p $377-$ 428, 1963.

(Fu3) H. FURSTENBERG - Random walk and discrete subgroups of Lie groups, Advances in Probability and related topics (Ed. P. Ney) vol. 1, p 1-63, M. Dekker, 1971.

(Fu4) H. FURSTENBERG - Boundary, theory and stochastic processes on homogeneous space, Proc. Symp. Pure Math. 36, p 193-229, 1972.

(Go-G) I. GOLDCHEID- Y. GUIVARC'H - Zariski closure and the dimension of the Gaussian law of the product of random matrices, Prob. theory related fields, 105, p 109-142, 1996.

(Go-M-P) I. GoldCheid, S.A. Molcanov, L.A. PAstur - A pure point spectrum of the one-dimensional Schroëdinger operators, Funct. Anal. Appl. 11, p 1-10, 1977.

(Go-M) I. GoldChEID, G.A. MARGULIS - Lyapunov indices of a product of random matrices, Russian Math. Surveys 44-5, p 11-81, 1989.

(Gree) GREENLEAF - Invariant means on topological groups, Van Nostrand, New York, 1969.

(Gren) GRENANDER - Probabilities on algebraic structures, Wiley, 1963.

(Gri) GRIGORTCHUK - The growth degrees of finitely generated groups and the theory of invariant means, Math. USSR Izv 25, p 259-300, 1985.

(Gri-Dlh) GRIGORTCHUK, DE LA HARPE - On problems related to growth, entropy and spectrum in group theory, Journal of dynamical and control systems $3, \mathrm{n}^{\circ} 1, \mathrm{p} 55-89,1997$.

(Gro) M. GROMOV - Groups of polynomial growth and expanding maps, Publ. Math. I.H.E.S., $n^{\circ}$ 53, p 53-78, 1981.

(Gu-H) Y. GUIVARC'H, J. HARDY - Théorèmes limites pour une classe de chaînes de Markov et applications aux difféomorphismes d'Anosov, Ann. I.H.P. $24, \mathrm{n}^{\circ} 1, \mathrm{p} 73-98,1988$.

(Gu-J-T) Y. GUIVARC'H, JI, J.C. TAYLOR - Compactifications of symmetric spaces, Progress in Math., Birkhaüser, 1998.

(Gu-K-R) Y. GUIVARC'H, M. KEANE, B. RoYNETTE - Marches aléatoires sur les groupes de Lie, Lecture Notes in Math. 624, Springer, 1977. 
(Gu-R1) Y. GUIVARC'H, A. RAUGI - Frontières de Furstenberg, propriétés de contraction et théorèmes de convergence, Z. Wahr 89, p 187-242, 1985.

(Gu-R2) Y. GUIVARC'H, A. RAUGI - Products of random matrices and convergence theorems, Contemraryt Math. 50 A.M.S., p 31-54, 1986.

(Gu1) Y. GUIVARC'H - Croissance polynomiale et périodes des fonctions harmoniques, Bull. S.M.F. 101, p 333-379, 1973.

(Gu2) Y. GUIVARC'H - Une loi des grands nombres pour les groupes de Lie, Astérisque 74, p 47-98, 1980.

(Gu3) Y. GUIVARC'H - Sur les exposants de Liapunoff des marches aléatoires, C.R.A.S. 292, p 327-329, 1981.

(Gu4) Y. GUIVARC'H - Produits de matrices aléatoires et applications aux sousgroupes du groupe linéaire, Ergod. Th. and Dyn. Syst. 10, p 483-512, 1990.

(Gu5) Y. GUIVARC'H -Quelques propriétés asymptotiques des produits de matrices aléatoires, Lecture Notes in math. 774, p 176-250, Springer, 1980.

(Gu6) Y. GUIVARC'H - Marches aléatoires sur les groupes et problèmes connexes, Publications des séminaires de l'université de Rennes, 1993.

(H) HADAMARD - Sur le battage des cartes et ses relations avec la mécanique statistique, Oeuvres t. 4, p 2071-2077, Éditions du C.N.R.S., 1968.

(He-S) HEBISH, L. SALOFF-CoSTE - Gaussian estimates for Markov chains and random walks on groups, à paraître.

(K) KAC - Probability and related topics in physical sciences, Interscience publishers, 1959.

(Ka-V) V.A. KAIMANOVICH, A.M. VERSHIK - Random walks on discrete groups : boundary and entropy, Annals of prob. vol. $11 \mathrm{n}^{\circ} 3, \mathrm{p} 457-490,1983$.

(Ka1) V.A. KAIMANOVICH - An entropy criterion for maximality of the boundary of random walks on discrete groups, Sov. Math. Dokl vol. 31, $\mathrm{n}^{\circ} 1, \mathrm{p}$ 193-197, 1985.

(Ka2) V.A. KAIMANOVICH - Boundaries of invariant Markov operators : the identification problem, Lecture Note series of London Math. Soc. 228, p 127176, 1996.

(Kaw-I) KAWADA-ITO - On the probability distribution on a compact group I, Proc. Phys. Math. Soc. Japan 22, p 226-278, 1940.

(Ke1) H. KESTEN - Symmetric random walks on groups, TAMS 92, p 336-354, 1959.

(Ke2) H. KESTEN - The Martin boundary of recurrent random walks on countable groups, Proc. Th. Berkeley Symp. on Math. Statistics and Probability t. II, p 51-74, 1967. 
(Ke3) H. KESTEN - Random difference equations and renewal theory for products of random matrices, Acta Math. 131, p 208-248, 1973.

(Ko) KORANYI - A survey of harmonic functions on symmetric spaces, Proceedings of Symposia in Pure Math. 35, I, p 323-344, 1979.

(L) LEVY - Processus stochastiques et mouvement brownien, 2 ème ed. Gauthier-Villars, Paris 1965.

(LeD) F. LE DRAPPIER - Quelques propriétés des exposants caractéristiques, Lecture Notes in Math. 1097, p 305-396, Springer, 1984.

(LeP) E. LE PAGE - Théorèmes limites pour les produits de matrices aléatoires, Lecture Notes in math. 928, p 258-303, Springer 1982.

(Let) LETCHIKOV - Products of unimodular independant random matrices, Russian Math. Surveys 51, n 1, p 49-96, 1996.

(Li-P) LIN-PINCHOVER - Manifolds with Group Actions and Elliptic Operators, Memoirs A.M.S. 112, $\mathrm{n}^{\circ}$ 540, 1994.

(Lo) LOSERT - On the structure of groups with polynomial growth, Math. Zeit. 196, p 109-117, 1987.

(Lu-P-S) A. LUBotsKy, R. PHILliPs, P. SARNAK - Hecke operators and distributing points on the sphere I, CPAM 39, p 149-186, 1987.

(Lu) LUBOTSKY - Discrete groups, expanding graphs and invariant measures, Progress in Math., Birkhaüser, 1994.

(Ly-S) T. LYONS, D. SULLIVAN - Function theory, random paths and covering spaces, Journal of Differential Geometry 19, p 299-323, 1984.

(Ly) T. LYONS - A simple criterion for transience of a reversible Markov chain, Ann. Prob. 11, p 393-402, 1983.

(Mi) MILNOR - A note on curvature and fondamental group, J. Diff. Geometry $2, \mathrm{p} 447-450,1968$.

(N-W) NASH-WILLIAMS - Random walks and electrical currents in networks, Proc. Cambridge Phil. Soc. 55, p 181-194, 1959.

(Ne) NEVo - Pointwise ergodic theorems for radial averages on simple Lie groups I, Duke Journal of Maths 76, $\mathrm{n}^{\circ} 1, \mathrm{p}$ 113-140, 1994.

(O) OSELEDETS - A multiplicative ergodic theorem Lyapunov : characteristic exponents for dynamical systems, Trans. Moscow Math. Soc. (A.M.S.) 19, p 197-231, 1968.

(P) PoINCARÉ - Calcul des Probabilités, 2ème ed., Gauthier-Villars, Paris, 1912.

(Pi) PIER - Amenable locally compact groups, Wiley New York, 1984. 
(Ra) A. RAUGI - Fonctions harmoniques et théorèmes limites pour les marches aléatoires sur les groupes de Lie, Bull. S.M.F. mém. 54, 1977.

(Ro) J. ROSENBLATT - Ergodicity and mixing of random walks on locally compact groups, Math. Ann. 257, p 31-42, 1981.

(Sa) SARNAK - Some applications of modular forms, Cambridge tracts in Math., 1990.

(So) SOARDI - Potential theory on infinite networks, Springer Lecture Notes 1590, 1994.

(Sp) F. SPITZER - Principles of random walks, Van Nostrand, 1964.

(Ts) TSUJI - Potential theory in modern function theory, Tokyo Maruzen, 1959.

(Tu1) TUTUBALIN - Some theorems of the type of the strong law of large numbers, Theory of Proba and its applications 14, p 313-319, 1969.

(Tu2) TUTUBALIN - A central limit theorem for products of random matrices and some of its applications, Symposia Math. vol. 21, Academic Press, p 101-116, 1977.

(V-S-C) N.Th. VARopoulos, L. SAloff-Coste, T. CoulhoN - Analysis and Geometry on groups, Cambridge University Press, 1991.

(V1) N. TH. VAROPOULOS - Analysis on Lie groups, Rev. Math. Iberoamericana 12, $\mathrm{n}^{\circ} 3, \mathrm{p}$ 791-917, 1996.

(V2) N. TH. VAROPOULOS - Information theory and harmonic functions, Bull Sc. Math. 110, p 347-389, 1986.

(V3) N. TH. VAROPOULOS - Théorie du potentiel sur les groupes et les variétés, CRAS sér. I 302, p. 203-205, 1986.

(Vi) VIRTSER - Central limit theorem for semi-simple Lie groups, Theory Proba Appl. 15, p 667-687, 1990.

(W) WEYL - The method of orthogonal projections in potential theory, Duke math. Journal 7, p 411-444, 1940.

(Wo) Woess - Random walks on infinite graphs and groups. A survey on selected topics, Bull. London Math. Soc. 26, p 1-60, 1994.

(Zi) R. ZIMMER - Ergocic theory and semi-simple groups, Birkhaüser Boston, 1984. 